\title{
Apport expérimental de la méthode de compactage statique des sols au laboratoire
}

\author{
Jean-François Serratrice ${ }^{\star}$ \\ Cerema Méditerranée, 30 rue Albert Einstein CS 70499, 13593 Aix-en-Provence, France
}

\begin{abstract}
Résumé - Une méthode de compactage statique a été mise en œuvre pour compacter des sols de diverses natures dans différentes conditions d'état, ou des sols traités, en vue de réaliser des essais de laboratoire. La méthode a été enrichie avec l'enregistrement des courbes donnant l'effort de compactage en fonction du tassement du sol. Ces courbes permettent de construire une surface d'état qui donne la déformation volumique du sol en fonction de la charge pendant son compactage à différentes teneurs en eau. La première partie de l'article est consacrée à une description de la compressibilité des sols dans diverses situations analogues où les sols se caractérisent par une grande porosité. La seconde partie s'adresse aux aspects expérimentaux du compactage statique, avec la description de la procédure mise en œuvre et des sols testés. Des exemples de surfaces d'état puis de courbes de compression statique sont présentés et commentés ensuite. Les paramètres qui sont identifiés sur ces courbes pour les caractériser sont définis. La dernière partie propose une récapitulation de ces paramètres, en lien avec la teneur en eau de compactage et d'autres propriétés des sols étudiés. L'exploitation de cette phase préliminaire de compactage statique présente l'intérêt de fournir des données complémentaires pour l'étude des sols compactés au laboratoire.
\end{abstract}

Mots clés : sols fins / compactage / courbe de compression / surface d'état / compressibilité

\begin{abstract}
Experimental contribution of the static soil compaction method in the laboratory. A static compaction method has been used to compact soils of various kinds under different state conditions, or treated soils, in order to carry out laboratory tests. The method was enhanced by monitoring the curves giving the compaction force as a function of soil settlement. These curves make it possible to build a state surface which gives the volumetric strain of the soil versus stress during compaction at different moisture contents. The first part of the paper is devoted to a description of the compressibility of soils in various analogous situations where soils are characterized by high porosity. The second part deals with the experimental aspects of static compaction, including the description of the procedure used and the soils tested. Examples of state surfaces and then static compression curves are presented and discussed. The parameters that are identified on these curves to describe them are defined. The last part proposes a summary of these parameters, in relation to the compaction water content and other properties of the soils. The purpose of this preliminary phase of static compaction is to provide complementary data to study compacted soils in the laboratory.
\end{abstract}

Keywords: fine soils / compaction / compression curve / state surface / compressibility

\section{Introduction}

Pour des raisons pratiques et économiques, le compactage des sols occupe une place centrale parmi les procédés de construction des ouvrages en terre. Le sol, initialement déstructuré depuis sa zone d'emprunt, se voit densifier pour offrir des caractéristiques mécaniques et hydrauliques spécifiques au sein de l'ouvrage en terre. L'accroissement de la raideur et de la résistance du sol compacté, mais aussi

\footnotetext{
$\bar{\star}$ Auteur de correspondance :

jean-francois.serratrice@cerema.fr
}

l'abaissement de sa perméabilité et de son érodabilité, vont de pair avec la réduction de la porosité du sol initialement foisonné.

En pratique, le compactage des sols en vue d'édifier des remblais s'effectue en appliquant des règles fondées sur l'expérience passée. L'essai Proctor constitue la référence universelle en la matière. Mais les règles se déclinent de façons différentes selon les pays. En Europe, la réalisation des terrassements fait référence à la norme NFEN 16907 (AFNOR, 2018). Toutes ces règles, qui sont destinées à l'exécution des terrassements, ne permettent pas de rendre compte du comportement mécanique des sols compactés une fois mis en place pour constituer l'ouvrage en terre, dans les 
conditions de chargement et environnementales attendues. Le recueil des propriétés mécaniques de déformabilité et de résistance ou les propriétés hydrauliques du sol compacté s'effectue à l'aide d'essais oedométriques ou d'essais triaxiaux au laboratoire. Au préalable et afin de réaliser ces essais, il est nécessaire de compacter des éprouvettes dont les réponses devront être aussi représentatives que possible du comportement du sol compacté au cœur de l'ouvrage.

Le compactage dynamique à la dame, de type Proctor ou dérivé, est utilisé pour reconstituer ces éprouvettes (Fleureau et Kheirbek-Saoud, 1992; Ferber et al., 2008). Mais le compactage statique est privilégié pour sa sensibilité et sa capacité à fournir des éprouvettes homogènes avec une bonne répétabilité (Bell, 1977; Camapum de Carvalho et al., 1987; Moussaï et al., 1993; Venkatarama Reddy et Jagadish, 1993 ; Delage et al., 1996; Mesbah et al., 1999; Tarantino et De Col, 2008; Saffit-Hdadi et al., 2009; Saad et al., 2012; Zhemchuzhnikov et al., 2015; Islam et Kodikara, 2016; Sharma et al., 2016; Wang et al., 2017). Les deux techniques coexistent depuis leur origine et ont donné lieu à plusieurs études comparatives au laboratoire (Bell, 1977; Sivakumar et Wheeler, 2000; Hafez et al., 2010; Crispim et al., 2011; Doris-Asmani et al., 2011; Wang et al., 2011; Ekwue et al., 2014; Yaghoubi et al., 2018). Parallèlement, une littérature abondante a été consacrée à la caractérisation des courbes de compactage des sols (courbes Proctor) sous la forme de surfaces d'état reliant la teneur en eau, la densité et l'énergie de compactage (Joslin, 1959; Pandian et al., 1997; Horpibulsuk et al., 2008; Horpibulsuk et al., 2013; Al-Badran et Schanz, 2014). Enfin, dès les années 1950 et sur de telles bases, les études ont permis de mettre en relation les propriétés mécaniques et hydrauliques des sols compactés avec leurs caractéristiques d'état (Seed, 1954 ; Leonards, 1955 ; Lambe, 1958; Seed et Chan, 1959).

Cette étude présente des données expérimentales recueillies pendant le compactage statique des éprouvettes destinées à la réalisation d'essais de laboratoire pour mesurer les propriétés mécaniques de nombreux sols compactés. Ces données ont été accumulées au fil du temps dans le cadre de projets d'ouvrages ou de programmes de recherche, sur des sols de natures diverses et compactés dans différentes conditions d'état. Le compactage statique est effectué en appliquant la méthode préconisée par Camapum de Carvalho et al. (1987). La densification du sol humide s'effectue par compression dans un moule. La méthode a été enrichie avec l'enregistrement de la courbe qui lie l'effort de compression au tassement du sol, en considérant que le compactage constitue l'histoire mécanique du sol compacté avant qu'il soit soumis à un essai triaxial ou un essai oedométrique. Sur la base d'essais faciles à mettre en œuvre et de la mesure de caractéristiques élémentaires (teneur en eau, indice des vides) ces courbes de compression statique procurent une description de la déformation volumique du sol, à partir de son état foisonné jusqu'à son état compacté, et fournissent des caractéristiques quantifiées (compacité initiale et compressibilité du sol foisonné, contrainte statique de compactage).

En s'appuyant sur ce recueil de données, l'étude propose de tirer parti des courbes de compression statique pour décrire le plan de compactage d'un sol ainsi densifié, en considérant que ces courbes contiennent déjà des indications sur les propriétés du sol compacté et son comportement pendant les chargements ultérieurs. Dans ce but, l'enregistrement des courbes de compression statique sert à construire une surface d'état qui relie la teneur en eau, l'indice des vides et la contrainte statique. Les propriétés mécaniques et hydrauliques qui sont mesurées ensuite sont pointées sur cette surface d'état (Moussaï et al., 1993 ; Venkatarama Reddy et Jagadish, 1993 ; Serratrice, 1995a, b, 2013; Tarantino et De Col, 2008; Al-Badran et Schanz, 2014; Sharma et al., 2016). Récemment, Kodikara (2012) a proposé un cadre simple et pratique fondé sur une telle surface pour expliquer et prédire les chemins d'état pendant le compactage des sols en condition oedométrique. D'autres développements ont suivi sur ce thème, qui montrent la pertinence de cette approche pour expliquer des mécanismes de reprise de compactage, d'effondrement sous imbibition, ou de gonflement des sols compactés (Islam et Kodikara, 2016; Kieu et Mahler, 2018).

Parmi l'ensemble des données recueillies, quelques courbes de compression statique sont présentées dans la suite. Elles ont été enregistrées pendant le compactage d'une dizaine de sols dans différents états foisonnés et traités pour certains d'entre eux. La diversité des réponses observées appelle à caractériser ces courbes par des paramètres représentatifs. Dans le but de définir ces paramètres, une analogie est recherchée avec les courbes de compressibilité affichées par les argiles sensibles, les sols compactés ou les sables, d'après des données de la littérature. Ces rappels bibliographiques font l'objet d'un premier paragraphe qui indique les démarches proposées par les auteurs pour caractériser les courbes de compressibilité des sols dans ces différents domaines. Une attention particulière est portée aux effets de structure et leur évolution, pour des sols qui se caractérisent par une grande porosité.

La section suivante est consacrée à la description de la méthode de compactage statique, puis à la présentation des sols testés et leurs propriétés physiques. Les paramètres proposés pour caractériser les courbes de compression statique sont définis. Ces courbes sont présentées au paragraphe trois. Les deux premiers exemples sont tirés de plans d'expériences élargis, qui ont permis de construire les surfaces d'état de compactage des deux sols testés et auxquelles appartiennent les courbes de compression statique. Les exemples suivants, recueillis dans le cadre de projets d'ouvrages, ne comptent que quelques courbes de compression statique enregistrées à partir de différents états foisonnés. Les paramètres de compressibilité sont identifiés sur les courbes expérimentales. La dernière section propose une récapitulation de ces paramètres, en lien avec les propriétés physiques des sols testés, leurs caractéristiques d'état ou leur résistance au cisaillement. Les mécanismes de compression des sols foisonnés sont interprétés par analogie avec les réponses des argiles sensibles et des sables. La discussion porte alors sur le caractère discriminant des courbes de compression statique et l'intérêt qu'elles présentent en pratique pour décrire le compactage des sols au laboratoire.

\section{Compressibilités de différents types de sols}

\subsection{Compressibilité des argiles sensibles saturées}

La compressibilité des argiles saturées s'interprète par la relation classique de Terzaghi (1927), où l'indice des vides $e$ 


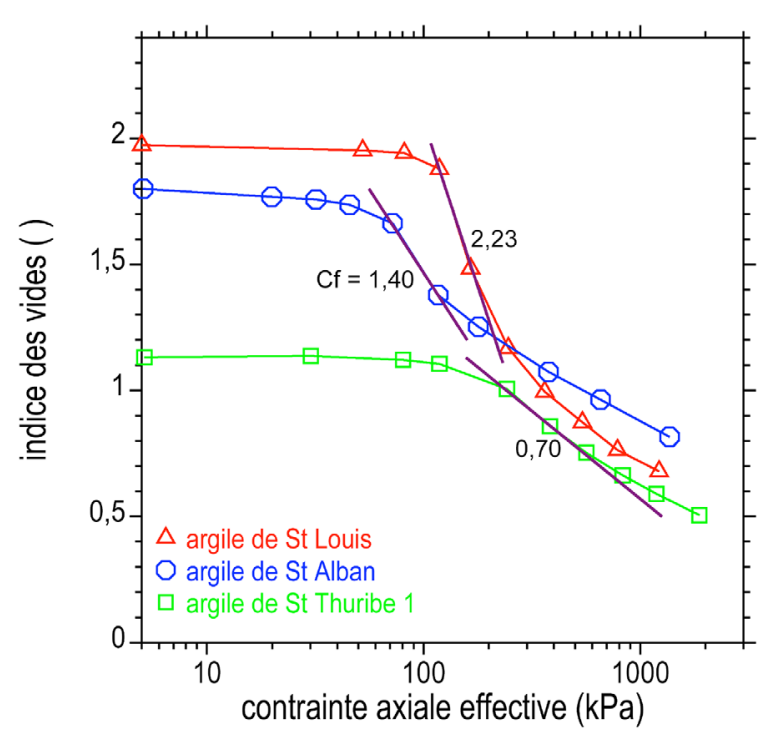

Fig. 1. Courbes de compressibilité de trois argiles sensibles de l'Est du Canada. D'après Delage (2010).

Fig. 1. Oedometer compression curves of three Eastern Canada sensitive clays.

diminue proportionnellement au logarithme de la contrainte axiale effective $\sigma_{a}^{\prime}$ pendant un chargement oedométrique monotone croissant au-delà de $\sigma^{\prime}{ }_{a c}$ :

$$
e=e_{0}-C_{c} \log \left(\sigma_{a}^{\prime} / \sigma_{a c}^{\prime}\right) \quad \sigma_{a}^{\prime}>\sigma_{a c}^{\prime}
$$

et où l'indice des vides $e_{0}$ et le coefficient de compressibilité $C_{c}=\Delta e / \Delta \log \left(\sigma_{a}^{\prime}\right)$ sont à identifier sur les courbes d'essais au laboratoire. Depuis Skempton et Jones (1944), nombreuses sont les corrélations qui ont été établies entre $C_{c}$ et les propriétés physiques des sols (Azzouz et al., 1976; Nagaraja et Srinivasa Murthy, 1983; Yoon et al., 2004; Kumar et al., 2016 ; et d'autres). Ces relations empiriques revêtent souvent un caractère régional. La limite de liquidité $w_{L}$ compte parmi les propriétés les mieux corrélées à la pente $C_{c}$, les argiles plastiques étant plus compressibles en général que les argiles peu plastiques.

La loi empirique (1) s'applique tout particulièrement aux argiles remaniées dans une large plage de contraintes effectives ( $\sigma^{\prime}$ ac vaut quelques kilopascals). Au contraire, les argiles naturelles récentes ne répondent à cette loi qu'au-delà de leur pression de préconsolidation $\sigma_{a p}^{\prime}$. En dessous de $\sigma_{a p}^{\prime}$, le régime de déformation de l'argile est pseudo-élastique. Dans son état naturel sous la contrainte $\sigma_{a p}^{\prime}$, une argile possède un indice des vides plus grand que dans son état remanié sous cette même pression (Burland, 1990; Liu et Carter, 1999). Cela tient aux effets de structure acquis pendant le processus de dépôt et post-dépôt de l'argile (Casagrande, 1932; Skempton et Northey, 1952; Leroueil et al., 1979; Leroueil et Vaughan, 1990 ; Cotecchia et Chandler, 2000). Ces effets sont accentués dans les argiles sensibles dont les courbes de compressibilité oedométrique $\left(\log \left(\sigma_{a}^{\prime}\right), e\right)$ font apparaître trois étapes successives. Après un palier pseudo-élastique de faible compressibilité à basse pression, un régime de transition apparaît, marqué par un seuil $\sigma_{\text {ay }}^{\prime}$, puis une forte compressibilité. La courbe se prolonge ensuite par une portion linéaire de pente comparable à celle de l'argile remaniée. À titre d'exemple, la figure 1 montre ces trois étapes de compressibilité pour trois argiles sensibles (Delage, 2010). Hong et al. (2012) évoquent trois régimes successifs de compressibilité le long des courbes, un régime pseudo-élastique avant le seuil, un régime de transition et un régime post-transition. Les pentes maximales $C_{f}=\max \left[\Delta e / \Delta \log \left(\sigma_{a}^{\prime}\right)\right]$ identifiées pendant la phase de transition des trois argiles sensibles sont indiquées dans la figure 1.

Ce grand contraste de compressibilité après le seuil marque la perte progressive des effets de structure de l'argile sensible (Leroueil et Vaughan, 1990; Schmertmann, 1991; Liu et Carter, 1999, 2000; Low et al., 2008; Yang et al., 2014). À partir de prélèvements dans divers sites de l'est du Canada, Delage (2010) montre que les argiles sensibles possèdent une microstructure composée d'agrégats. En compression oedométrique, la diminution du volume s'opère par une réduction progressive des vides inter-agrégats en commençant par les plus grands, pour se propager ensuite aux pores de plus en plus petits. De même, d'autres études ont été menées dans ce sens au moyen d'analyses microstructurales (Hattab et al., 2010; Hattab et al., 2013; Cotecchia et al., 2016). Pendant le chargement, la réduction des vides s'accompagne de la dégradation progressive des liaisons inter-agrégats.

Liu et Carter $(1999,2000)$ ont proposé de décrire la compression des argiles structurées pendant la phase de transition après le seuil $\left(\sigma^{\prime}{ }_{a}>\sigma^{\prime}\right.$ ay $)$ par une hyperbole située au-dessus de la ligne de compression vierge dans le plan (log $\left.\left(\sigma_{a}^{\prime}\right), e\right)$ et qui prend cette ligne pour une asymptote à haute pression. Avant le seuil $\left(\sigma^{\prime}{ }_{a}<\sigma^{\prime}{ }_{a y}\right)$, la phase pseudo-élastique de l'argile est caractérisée par la droite de pente $C_{S}$ (coefficient de gonflement). La courbe commence au point $\left(\sigma^{\prime}\right.$ ay, $\left.e_{y}\right)$, où l'écart $\Delta e_{y}$ est maximal entre $e_{y}$ et l'indice des vides correspondant sur la ligne de compression vierge. De même, la pente de la courbe est maximale en ce point anguleux. Dans la formulation, cette pente dépend d'un exposant $b$ qualifié d'indice de déstructuration par compression. Les pentes $C_{f}$ reportées dans la figure 1 donnent un ordre de grandeur de cette pente maximale. Liu et Horpibulsuk (2015) et Qian et al. (2016) complètent cette formulation pour donner une description plus large du processus de déstructuration des argiles naturelles par compression, d'après les courbes de compressibilité.

Ces recherches ont montré aussi que la compressibilité des argiles remaniées dépend de leur teneur en eau initiale (Cerato et Lutenegger, 2004; Hong et al., 2012; Zeng et al., 2015). Dans un sol, en partant d'indices des vides plus grands aux fortes teneurs en eau, les courbes de compressibilité se situent au-dessus de celles enregistrées à teneurs en eau plus faibles. Ces courbes se rejoignent ensuite à haute pression. Qian et al. (2016) proposent une méthode pour tenir compte de cet effet afin d'évaluer la part des vides supplémentaires que possède une argile sensible par rapport à l'une de ses configurations remaniées.

\subsection{Compressibilité des argiles foisonnées non saturées}

La figure 2 compare les courbes de compressibilité oedométrique d'une argile limoneuse initialement foisonnée et préparée à différentes teneurs en eau initiales $w_{0}$, d'après 


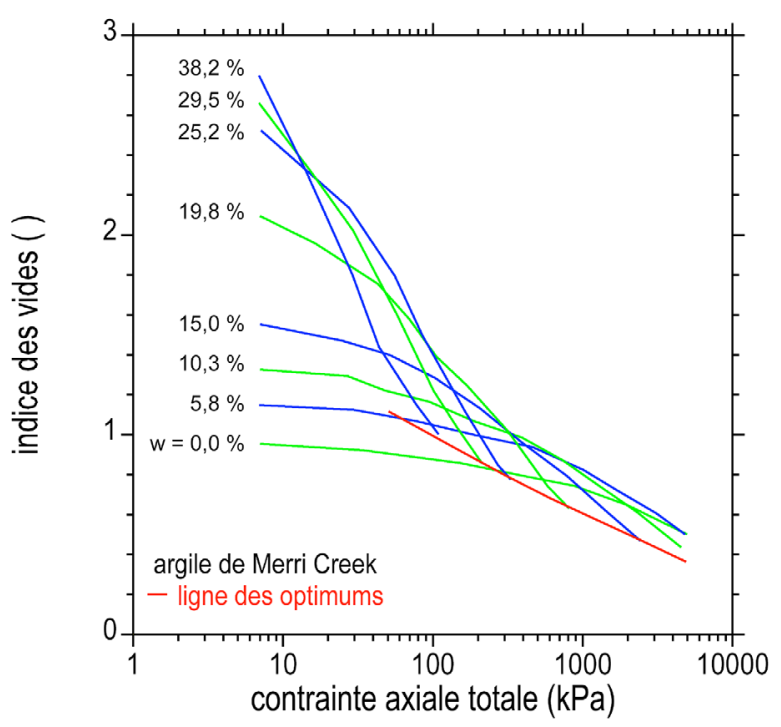

Fig. 2. Courbes de compression d'une argile plastique préparée à différentes teneurs en eau initiales. D'après Islam et Kodikara (2016). Fig. 2. Compression curves of a plastic clay for different initial moisture contents.

Islam et Kodikara (2016). Les courbes sont représentées dans le plan $\left(\log \left(\sigma_{a}\right), e\right)$ où $\sigma_{a}$ est la contrainte axiale totale et $e$ l'indice des vides. Le compactage statique est effectué à teneur en eau constante, à vitesse lente, à partir de $7 \mathrm{kPa}$, jusqu'à atteindre une compacité correspondant à la ligne des optimums Proctor ou la contrainte maximale de $7000 \mathrm{kPa}$. Pour les plus courantes, ces courbes de compression présentent un palier initial suivi par une étape de forte compressibilité. Le palier est réduit ou absent pour les états les plus lâches correspondants aux plus fortes teneurs en eau. Il se prolonge largement à haute pression pour les plus faibles teneurs en eau, qui sont associées aux états foisonnés les plus denses. Après un seuil $\left(\sigma_{a y}, e_{y}\right)$, qui marque le passage vers les fortes compressibilités, les pentes $\Delta e / \Delta \log \left(\sigma_{a}\right)$ des courbes $\left(\log \left(\sigma_{a}\right), e\right)$ varient avec $\sigma_{a}$. Mais elles dépendent aussi de la teneur en eau $w_{0}$. Les pentes maximales $C_{f}$ de ces courbes augmentent avec $w_{0}$, en allant de 0,38 à 1,82 dans la figure 2 . En contrepartie, l'ordonnée $e_{y}$ du seuil diminue rapidement. De nombreuses observations concernant le compactage statique des argiles vont dans ce sens (Lin et Lowell, 1981; Guérif, 1982; Venkatarama Reddy et Jagadish, 1993; Serratrice, 1995a, b, 2013; Mesbah et al., 1999; Koliji et al., 2009; Mun et McCartney, 2015 ; Kieu et Mahler, 2018).

Après avoir atteint une compacité correspondant à la ligne des optimums Proctor, l'état du sol tend vers la saturation. La poursuite de la densification en mode statique dépend des conditions de drainage du sol. Sans drainage (teneur en eau maintenue constante), le chargement ne produit qu'une petite compression supplémentaire du sol, accompagnée par la génération de pressions dans l'air occlus et dans l'eau interstitielle (Hilf, 1956 ; Lambe, 1961 ; Fredlund et Rahardjo, 1993 ; Vaughan, 2003). Au contraire, si le drainage du sol est possible, la compression du sol se poursuit avec la diminution de la teneur en eau et de l'indice des vides. La courbe (log $\left.\left(\sigma_{a}\right), e\right)$ suit alors un chemin descendant quasiment parallèle à la courbe de compressibilité de l'argile remaniée saturée de pente $C_{c}$ ( $w$ et $e$ diminuent).
Les sols argileux possèdent des indices des vides plus grands dans leurs états foisonnés que dans leurs états saturés. Cela confère au sol foisonné un régime équivalent à celui d'une préconsolidation, avec un palier pseudo-élastique et un seuil $\sigma_{a y}$. Mais, contrairement aux argiles naturelles saturées, les sols foisonnés sont effondrables (Lutenegger et Saber, 1988; Lawton et al., 1992; Sun et al., 2007; Leong et al., 2013). Ainsi, dans la figure 2 et à partir d'un état foisonné humide $\left(w_{0}>10 \%\right)$, l'imbibition de l'argile non saturée en un point d'une des courbes produirait un effondrement de sa structure. L'argile atteindrait alors un nouvel état proche de la courbe de compressibilité de l'argile remaniée saturée, sous cette même charge (Jennings et Burland, 1962; Leclercq et Verbrugge, 1985; Delage et Fry, 2000; Koliji et al., 2009; Burton et al., 2014 ; et d'autres). Parallèlement des études ont été menées à l'échelle microscopique pour montrer l'évolution des agrégats, des macro-pores et des micro-pores, qui constituent la structure des sols compactés, pendant les mécanismes de déformation (Delage et al., 1996; Cuisinier et Laloui, 2004 ; Ferber et al., 2008; Alonso et al., 2013).

Le rôle de la succion, différence entre les pressions de l'air et de l'eau dans les pores du sol non saturé, et celui du degré de saturation, rapport entre le volume d'eau et le volume des vides, ne peuvent pas être ignorés dans les mécanismes évoqués ci-dessus. Dans cette optique, nombreux sont les travaux expérimentaux qui ont été consacrés à l'étude du comportement mécanique des sols non saturés, et des sols compactés en particulier, pour décrire et quantifier le rôle de la succion et du degré de saturation (Biarez et al., 1987; Cui et Delage, 1996; Tarantino et De Col, 2008 ; Koliji et al., 2009; Sivakumar et Wheeler, 2000; Kodikara, 2012; Alonso et al., 2013; Lloret-Cabot et al., 2017; parmi d'autres). Dans ce cadre, les variations de volume observées le long des chemins isotropes ou oedométriques sont reproduites au moyen d'essais à succion contrôlée. L'effet d'une préconsolidation apparente augmente avec la succion (l'indice des vides initial foisonné et le seuil $\sigma_{a y}$ ). Si le palier pseudo-élastique initial semble peu dépendant de la succion, le seuil $\sigma_{a y}$ et la compressibilité $C_{f}$ qui fait suite en sont très dépendants (Cui et Delage, 1996; Alonso et al., 2013).

Cependant, en matière de déformabilité et de résistance des sols compactés en lien avec leur état, l'interprétation des essais en contraintes totales conserve un intérêt pratique. Ainsi, les faisceaux de courbes du type de la figure 2 permettent de construire une surface unique $\left(w, e, \log \left(\sigma_{a}\right)\right)$ donnant tous les états du sol pendant les compressions oedométriques monotones, depuis différents états foisonnés et dans ces conditions de mise en œuvre du compactage (Serratrice, 1995a, b; AlBadran et Schanz, 2014). L'analyse des courbes de compression statique, qui sera abordée plus bas, s'inscrit dans cette thématique. Cette démarche peut être étendue à d'autres modes de compactage et d'autres types de chargement-déchargement (Kodikara, 2012; Islam et Kodikara, 2016; Kodikara et al., 2016).

\subsection{Compressibilité des matériaux granulaires}

Beaucoup de sols contiennent des particules friables constituées par des roches tendres ou cataclasées ou altérées (calcaires bioclastiques, granites décomposés, par exemple). En cours de chargement, l'écrasement des particules marque la 


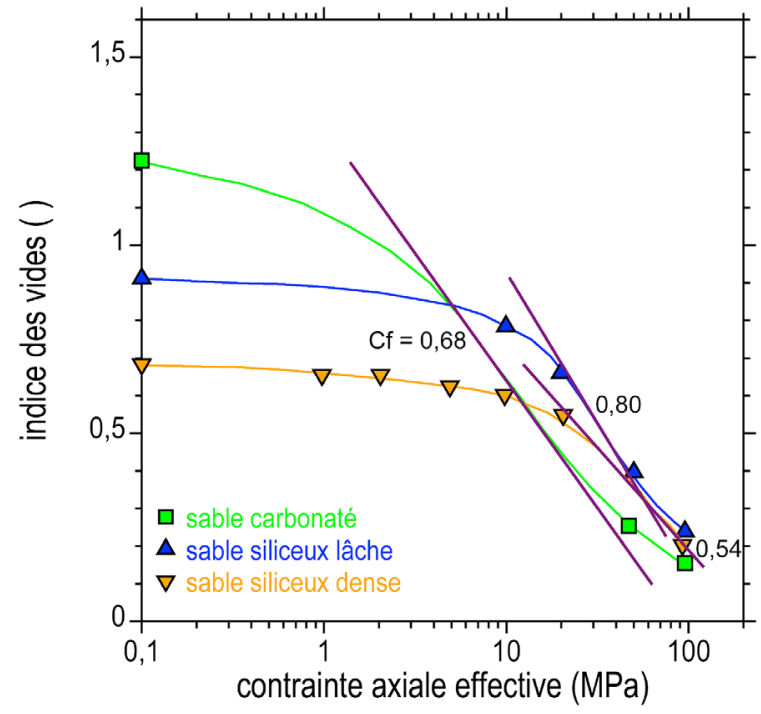

Fig. 3. Courbes de compression oedométrique de trois sables. D'après Golightly (1989), dans McDowell et Bolton (1998).

Fig. 3. One-dimensional compression plots for carbonate and silica sands.

réponse des sols qui exhibent des compressibilités fortes, des résistances réduites et des dilatances faibles. L'étude du comportement mécanique de ces terrains a été étendue progressivement à l'ensemble des sols granulaires pour rendre compte de l'effet de l'écrasement des particules pendant les chargements (Yamamuro et al., 1996 ; Biarez et Hicher, 1977; McDowell et Bolton, 1998; Nakata et al., 2001; McDowell, 2002). Dans ce cadre, de nombreuses études expérimentales ont été consacrées aux sables à granulométrie uniforme soumis à des très fortes contraintes en condition oedométrique ou isotrope (plusieurs centaines de mégapascals). Les réponses partagent une allure commune en termes de contrainte axiale effective $\sigma_{a}^{\prime}$ et d'indice des vides $e$. Les courbes de compressibilité oedométriques $\left(\log \left(\sigma_{a}^{\prime}\right), e\right)$ exprimées en échelles logarithmiques montrent un palier pseudo élastique de faible déformabilité suivi par un domaine de forte compressibilité (Fig. 3). Dans ce domaine, les coefficients de compressibilité atteignent des niveaux élevés, avec des pentes maximales $C_{f}$ souvent comprises entre 0,4 et 0,6 ou plus. Les deux domaines de déformabilité sont séparés par une zone intermédiaire courbée plus ou moins étendue et arrondie, qui marque les signes d'écrasement des grains. Un seuil d'abscisse $\sigma_{a y}^{\prime}$ caractérise cette zone intermédiaire. La distribution granulométrique du sable évolue au cours du chargement, avec la production d'éléments fins.

Dans les sables uniformes, le seuil d'écrouissage $\sigma_{a y}^{\prime}$ dépend de la dimension des grains (Nakata et al., 2001). Il augmente avec la diminution du diamètre des grains. Il diminue avec l'accroissement de l'indice des vides initial $e_{0}$. Les sables anguleux produisent un seuil $\sigma^{\prime}{ }_{a y}$ plus faible que les sables roulés, témoignant ainsi d'une initiation de l'écrasement de grains sous les faibles pressions. La transition est continue ensuite. Au contraire, les sables roulés produisent un seuil marqué sous les fortes pressions, suivi par une phase de grande compressibilité ( $C_{f}$ peut atteindre l'unité). Après ce fort tassement, la courbe de compressibilité tend à rejoindre celle du sable anguleux de même nature. De son côté, la nature minéralogique de la roche qui constitue les grains contrôle leur résistance à l'écrasement (Hagerty et al., 1993; Pestana et Whittle, 1995; McDowell et Bolton, 1998; Chuhan et al., 2003 ; Sheng et al., 2008 ; Dadda et al., 2015; Wu et al., 2016; De Bono et McDowell, 2017). Les grains les plus friables produisent les seuils $\sigma_{a y}^{\prime}$ les plus faibles, puis des transitions continues, contrairement aux grains de quartz. Enfin, parmi d'autres facteurs influents, les contraintes de cisaillement produites par les chargements triaxiaux accentuent les ruptures de grains, en comparaison des chargements isotropes ou oedométriques (Hicher et al., 1995; Daouadji et al., 2001; Sheng et al., 2008).

Les sables uniformes possèdent des vides qui favorisent les mécanismes de déplacement relatif et d'écrasement des grains. Au moyen d'essais oedométriques, Altuhafi et Coop (2011) ont montré que les ruptures de grains s'observent en plus grand nombre dans les sables mal gradués que dans les sables bien gradués. À la limite, trop rares, les ruptures de grains échappent à l'observation dans les sables très bien gradués. Mais, en dépendant de la densité initiale, de la distribution granulométrique des grains et de la proportion des particules friables, entre autres, les courbes de compressibilité (log $\left.\left(\sigma_{a}^{\prime}\right), e\right)$ ne convergent pas toujours vers une asymptote commune à très haute pression. Shipton et Coop (2012) testent des mélanges granulaires de différentes minéralogies et de différentes granulométries en conditions oedométriques pour étudier la transition de comportement entre les sables mal gradués et les sables bien gradués. Cette transition s'avère très sensible au changement de la nature des grains et aux petites variations des proportions entre les classes granulométriques. La convergence des courbes de compressibilité $\left(\log \left(\sigma_{a}^{\prime}\right), e\right)$ vers une asymptote commune est favorisée dans les mélanges contenant de fortes proportions de particules friables. Au-delà des tendances évoquées ici, il n'apparaît pas de lois explicites liant les compressibilités aux nombreux facteurs dont elles dépendent. Toutes ces observations ne s'appuient pas sur des analyses microstructurales détaillées comme dans les deux domaines examinés précédemment. Néanmoins, les résultats expérimentaux font état indirectement du rôle des vides et des contacts entre les grains et leur évolution sous les fortes pressions, à cette échelle.

\subsection{Pressage des poudres}

Le pressage des poudres constitue un procédé largement utilisé dans de nombreux secteurs des industries minéralières, métallurgiques, pharmaceutiques, aérospatiales, agroalimentaires, cosmétiques, et autres. Ce procédé est adapté à la fabrication de pièces de petites dimensions. Dans le domaine de la métallurgie des poudres, la matière est comprimée dans un moule (matrice) sous de très fortes pressions (plusieurs centaines de mégapascals) pour obtenir un matériau comprimé «à vert» ou «à cru » (green body). Le pressage (die pressing) confère au matériau une cohésion et une résistance. La « consolidation» du matériau peut être améliorée ensuite par le procédé du frittage qui consiste à appliquer un traitement thermique.

Le passage du matériau en poudre au matériau comprimé est étroitement lié aux propriétés plastiques des grains. L'optimisation des procédés a donné lieu à de nombreuses 
études expérimentales et théoriques dans le but d'améliorer les méthodes et la prévision des densités et des propriétés finales des produits (Chandler et al., 2008; Han et al., 2008; Bosi et al., 2013). L'empilement initial des grains joue un rôle prépondérant dans la qualité d'un corps cru, dans sa tenue mécanique et sa capacité de densification.

Les courbes contrainte-déformation $\left(\varepsilon_{a}, \sigma_{a}\right)$ présentent une allure exponentielle typique des chargements oedométriques. De nombreuses expressions empiriques ont été proposées pour décrire la densification des poudres par pressage. Walker (1923) propose l'une des premières expressions qui relie le volume relatif $V / V_{0}$ au logarithme de la pression appliquée $p$, de la forme, $V / V_{0}=a-b \ln (p)$, où $a$ et $b$ sont des paramètres à identifier sur la courbe expérimentale de compactage, $V$ le volume actuel et $V_{0}$ le volume initial. Selon Bal'shin (1938), une expression similaire peut être obtenue en supposant que l'énergie $\mathrm{d} W$ dissipée par frottement pendant le pressage pour produire la variation de volume irréversible $\mathrm{d} V$ est proportionnelle à l'incrément de pression $\mathrm{d} p(\mathrm{~d} W=p \mathrm{~d} V=\Lambda \mathrm{d} p$ où $\Lambda$ est un coefficient de proportionnalité). Dans les conditions d'un chargement oedométrique $\left(p=\sigma_{a}\right)$, l'intégration de cette expression aboutit à l'équation (1).

\subsection{Traits communs pour les sols}

La densification des sols concerne aussi d'autres domaines techniques. En agronomie, la compaction des sols joue en défaveur des cultures (réduction du volume des pores, du stockage de l'eau et des flux). Les études portent sur des sols peu compacts (foisonnés) dont les structures sont majoritairement constituées par des agrégats et un réseau de pores de différentes formes, dimensions et orientations (Keller et al., 2013; Menon et al., 2015). Les sols sont organiques. Ils sont parfois entrecoupés par des fissures de retrait. Mais les mêmes tendances se retrouvent, avec l'influence de la nature des particules sur leur foisonnement et leur pseudo-préconsolidation, mais aussi l'influence des matières organiques ou celle d'éléments grossiers (Rücknagel et al., 2016). Dans un état foisonné, les argiles sont plus lâches que les sables. Une teneur minimale en éléments grossiers s'avère nécessaire pour éviter la compaction des particules fines et augmenter la pression de préconsolidation du sol.

Dans le domaine géotechnique, les observations tirées de trois sources différentes, les argiles sensibles, les sols fins compactés et les sables, ont en commun de présenter un régime de préconsolidation réel ou équivalent se manifestant par un palier pseudo-élastique, puis un seuil au-delà duquel des fortes compressibilités sont observées pendant les chargements monotones oedométriques ou isotropes. Avec des pentes $\Delta e / \Delta \log \left(\sigma_{a}\right)$ dont les valeurs maximales $C_{f}$ sont de l'ordre de 0,5 et plus, les compressibilités sont bien supérieures aux coefficients $C_{c}$ mesurés habituellement sur les argiles naturelles non organiques. Mais les seuils de préconsolidation évoluent sur plusieurs ordres de grandeur des pressions. Les observations microscopiques confirment ces tendances. Ces terrains ont en commun des structures poreuses formées de grains, d'agrégats et de pores. La densification s'opère par fermeture de l'espace poreux, la contraction et la distorsion des agrégats, le réarrangement et l'écrasement des grains, la destruction des liaisons entre les grains. La densification se développe avec dissipation d'énergie par frottement et rupture.
En dépendant de la nature du sol, la loi logarithmique de compressibilité (1) s'applique par morceaux dans des intervalles plus ou moins limités de la gamme des contraintes exercées pendant les essais oedométriques courants. Formellement, elle n'est pas applicable à très faible pression (e devient infini) et à très forte pression ( $e$ devient négatif). Aussi, d'autres formulations empiriques ont été proposées afin de couvrir des gammes de contraintes plus étendues (JuarezBadillo, 1981; Chong et Santamarina, 2016). Il s'agit notamment du domaine du pressage des poudres ou celui des essais à très haute pression effectués sur les sols granulaires. La loi bi-logarithmique $\left(\log \left(\sigma^{\prime}{ }_{a}\right), \log (e)\right)$ est souvent mise en avant parmi ces formulations empiriques (Pestana et Whittle, 1995; Russell, 2011; Hong et al., 2012; Sharma et Ismail, 2016; De Bono et McDowell, 2017).

\section{Méthode expérimentale et matériaux étudiés}

\subsection{Compactage statique}

Dans la pratique courante au laboratoire, le compactage statique sert à reconstituer des éprouvettes, qui seront soumises ensuite à des essais mécaniques, afin de mesurer diverses caractéristiques parmi les propriétés de compressibilité, de gonflement, de perméabilité, de déformabilité et de résistance des sols compactés. Cela concerne aussi les sols traités par des liants ou des fibres, puis compactés. Toutefois, pour rester compatibles avec les appareillages standards de mécanique des sols, les éprouvettes possèdent des petites dimensions, ce qui restreint l'utilisation courante du procédé au compactage des sols fins (diamètres des éprouvettes compris entre 50 à $100 \mathrm{~mm})$.

Le compactage statique est privilégié pour sa sensibilité et sa capacité à fournir des éprouvettes homogènes avec une bonne répétabilité (Camapum de Carvalho et al., 1987; Moussaï et al., 1993; Cui et Delage, 1996; Mesbah et al., 1999; Doris-Asmani et al., 2011). Le compactage d'une éprouvette s'effectue dans un moule cylindrique rigide à l'aide d'une presse de compression. Le moule est conçu pour produire une éprouvette d'élancement final donné, à partir du sol foisonné. Les faibles élancements sont obtenus avec des cellules oedométriques (Tarantino et De Col, 2008; Koliji et al., 2009; Wang et al., 2017). Les moules d'élancement voisin de un sont les plus répandus (Moussaï et al., 1993; Venkatarama Reddy et Jagadish, 1993; Mesbah et al., 1999; Mun et McCartney, 2015; Islam et Kodikara, 2016). Enfin, d'autres montages fournissent des éprouvettes d'élancement deux pour essai triaxial, en une seule couche (Camapum de Carvalho et al., 1987 ; Saad et al., 2012 ; Hafez et al., 2010) ou plusieurs couches (Delage et al., 1996). Les parois des moules sont lubrifiées afin de réduire les frottements latéraux. La compression s'effectue entre des pistons flottants dans la plupart des cas. La compression est contrôlée en déplacement ou en effort, selon un rythme donné lent, ce qui en fait un processus statique. L'homogénéité du sol compacté peut être testée par pesées de tranches d'éprouvettes (Saad et al., 2012) ou par gamma-densitométrie (Camapum de Carvalho et al., 1987 ; Cui et Delage, 1996).

À l'exclusion des matériaux granulaires ou très frottants, la méthode de compactage statique permet de compacter les sols 
a)

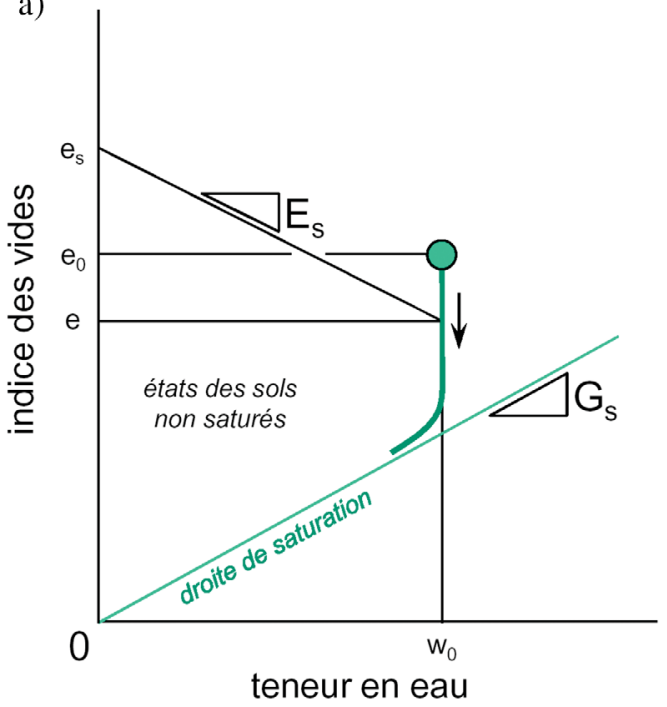

état initial foisonné non saturé $\left(w_{0}, e_{0}, \sigma_{a 0}\right)$ b)

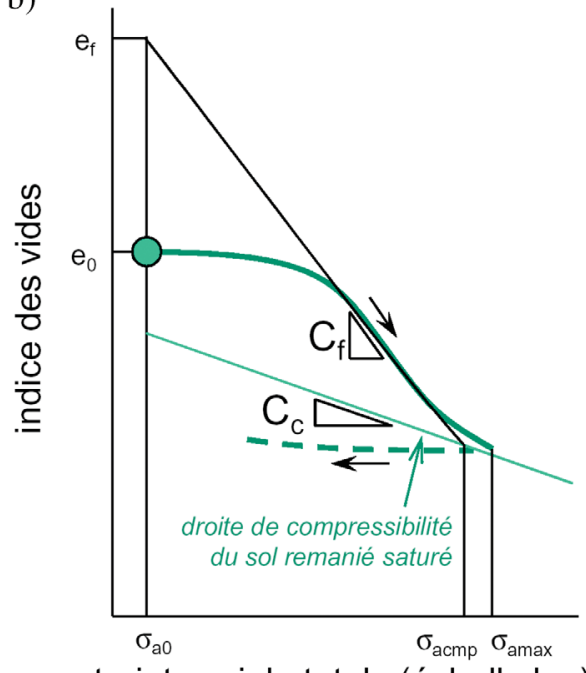

contrainte axiale totale (échelle log)

courbe de compression semi-statique

Fig. 4. Courbes de compression d'un sol: (a) dans le plan d'état $(w, e)$; (b) dans le plan de compressibilité $\left(\log \left(\sigma_{a}\right), e\right)$.

Fig. 4. Compression curves of a soil: $(a)$ in the state plane (w,e); (b) in the one-dimensional compression plane $\left(\log \left(\sigma_{a}\right), e\right)$.

fins dans une large gamme de teneurs en eau. Néanmoins, il est nécessaire de déployer de fortes contraintes de compactage dans les sols secs et le compactage des sols saturés n'est possible que si la consolidation du sol est autorisée. L'optimum Proctor normal et l'optimum Proctor modifié se trouvent au centre d'un domaine privilégié, ni trop sec, ni trop humide, qui correspond aux conditions de mise en œuvre les plus appropriées de la plupart des sols dans les applications courantes. Le compactage statique permet de couvrir ce domaine pour les sols fins.

Dans la suite, le compactage statique des sols au laboratoire est examiné à l'aide des trois variables principales : la teneur en eau $w$, l'indice des vides $e$ et la contrainte axiale totale $\sigma_{a}$ (Fig. 4). Le sol est initialement foisonné au point d'état $\left(w_{0}, e_{0}\right)$. Au plan $\left(\log \left(\sigma_{a}\right), e\right)$, où s'observe la compressibilité du sol, est associé le plan d'état $(w, e)$. Introduite par Terracina (1955; voir Andrei, 1977), l'utilisation du plan (w,e) présente l'avantage d'offrir une forme linéaire aux variations des principales caractéristiques d'état du sol. Ainsi, les courbes iso-degré de saturation sont des droites d'équation $e=\left(w \rho_{s}\right) /$ $\left(S_{r} \rho_{w}\right)$ où $\rho_{s}$ est la masse volumique des particules solides, $\rho_{w}$ la masse volumique de l'eau et $S_{r}$ le degré de saturation. L'équation s'écrit aussi :

$$
S_{r} e=G_{s} w
$$

où $G_{s}=\rho_{s} / \rho_{w}$ est la densité des particules solides. En particulier, la droite de saturation a pour équation $e=G_{s} \mathrm{~W}$ $\left(S_{r}=1\right)$. Les deux droites de pente $G_{s}$ et $C_{c}$ de la figure 4 représentent le mécanisme d'écrouissage de l'argile déstructurée saturée pendant un chargement lent drainé. Leur unicité constitue un trait fondamental du comportement des argiles.

L'utilisation d'une version équivalente au plan d'état $(w, e)$ est proposée par Kodikara (2012), qui suggère d'adopter l'indice des vides $e_{w}=G_{s} w$ comme variable représentant la teneur en eau. Cet indice des vides correspond à l'ordonnée du point d'abscisse $w$ sur la droite de saturation $e=w G_{s}$ du plan d'état $(w, e)$. Ainsi, les deux coordonnées du plan d'état $\left(e_{w}, e\right)$ sont homogènes à un indice des vides et la droite de saturation a pour pente l'unité. Cette part $e_{w}$ des vides est qualifiée par Ferber et al. (2008) d'indice des vides des agrégats. Dans un essai à teneur en eau constante à partir d'un état foisonné $\left(w_{0}, e_{0}\right)$, cette part des vides située sous la droite de saturation n'est pas réductible (Fig. $4 \mathrm{a}$ ). Seule, la différence $e-e_{w}$, dite inter-agrégats, l'est (part des vides située au-dessus de la droite de saturation).

Le choix de l'état du sol compacté, objectif du compactage statique, est défini par une densité et une teneur en eau. Les consignes de compactage sont données sous la forme du couple d'une teneur en eau et d'une masse volumique ( $\left.w_{\text {visée }}, \rho_{\text {dvisée }}\right)$. Sauf cas particuliers, ces consignes font référence à l'optimum Proctor normal du sol compacté (teneur en eau $w_{\mathrm{OPN}}$ et masse volumique sèche $\left.\rho_{\mathrm{dOPN}}\right)$. L'indice des vides $e_{\text {visé }}=\rho_{s} / \rho_{\text {dvisée }}-1$ est associé à la consigne de compactage. Depuis son état foisonné $\left(w_{0}, e_{0}\right)$, la densification du sol par compression monotone s'effectue en condition oedométrique dans un moule. L'enregistrement du tassement du sol et de l'effort de compression permet de décrire la variation de l'indice des vides en fonction de la contrainte totale axiale de compactage. Associées à la teneur en eau, les courbes de compression statique sont exprimées dans l'espace des trois variables $\left(w, e, \log \left(\sigma_{a}\right)\right)$, comme indiqué dans la figure 4 .

Dans le plan d'état $(w, e)$ de la figure $4 \mathrm{a}$, le chemin de compactage s'effectue à teneur en eau constante $w_{0}$, jusqu'à aboutir à un état proche de la saturation. L'indice des vides minimal qu'il est possible d'atteindre à teneur en eau constante est $e_{\text {inf }}=w_{\text {visée }} G_{s} / S_{\text {rinf }}$ où le degré de saturation $S_{\text {rinf }}$ est proche de l'unité (sol quasi saturé). Une compacité plus faible ne peut être obtenue $\left(e_{\text {visé }}<e_{\text {inf }}\right)$ que si le drainage du sol est possible, en allongeant la durée du chargement et en augmentant la charge axiale. Alors, la teneur en eau finale sera proche de $w_{\text {fin }}=e_{\text {visé }} S_{\text {rinf }} / G_{s}$, en admettant que la poursuite de la 
a)

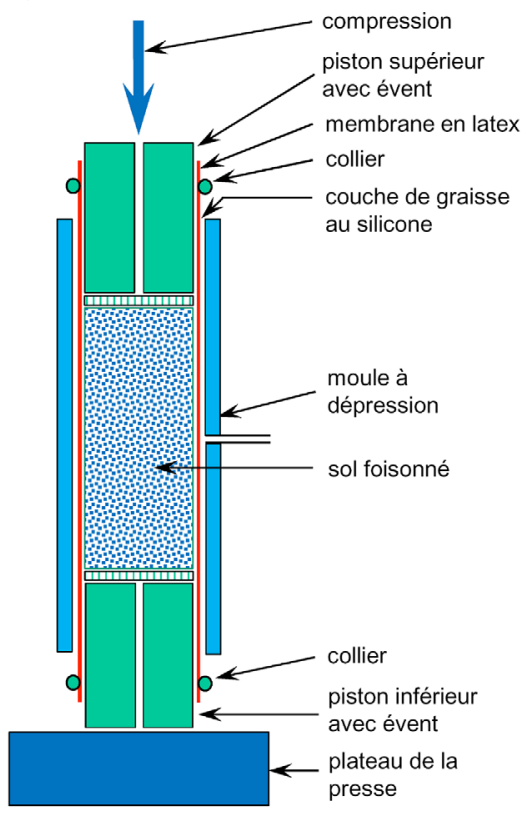

b)

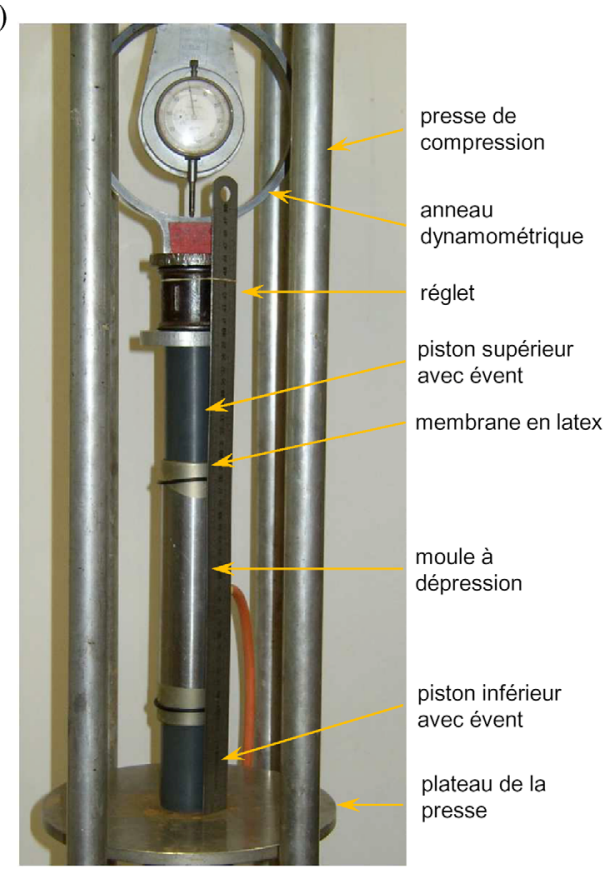

Fig. 5. Procédé de compactage statique d'un sol: (a) schéma du moule de compactage; (b) photo du bâti de chargement et du moule. Fig. 5. Method of soil compaction: (a) diagram of the mold; (b) photo of the loading frame and the mold.

densification s'effectue à degré de saturation constant $\left(w_{\text {fin }}\right.$ $<w_{\text {visée }}$ ). Le chemin d'état suit la courbe indiquée sur le graphique. Mais, dans la plupart des cas courants et notamment ceux situés sur la ligne des optimums Proctor, le point d'état reste sur la partie rectiligne du chemin. La figure $4 \mathrm{~b}$ montre la courbe de compression $\left(\log \left(\sigma_{a}\right), e\right)$ du sol foisonné, à partir de son état initial $\left(\sigma_{a 0}, e_{0}\right)$ jusqu'à la contrainte axiale maximale $\sigma_{a \max }$ qu'il est nécessaire d'appliquer pour atteindre l'état de compacté visé ( $\sigma_{a 0}=1 \mathrm{kPa}$ est la contrainte axiale unité). La courbe de compression statique se prolonge par la courbe de déchargement (en pointillés). La courbe de compression se situe au-dessus de la droite de compressibilité du sol remanié saturé de pente $C_{c}$. Elle possède une allure comparable à celle des courbes expérimentales présentées dans la section précédente. Elle peut être caractérisée par la pente maximale $C_{f}=\max \left[\Delta e / \Delta \log \left(\sigma_{a}\right)\right]$ de sa tangente dont l'équation est :

$$
e=e_{f}-C_{f} \log \left(\sigma_{a} / \sigma_{a 0}\right),
$$

et l'indice des vides $e_{f}$ (ordonnée à l'origine de la tangente). La contrainte axiale maximale $\sigma_{a c m p}$ correspond à l'abscisse du point où la tangente de pente $C_{f}$ rejoint la droite de pente $C_{c}$, pour un compactage à teneur en eau constante. Les paramètres $e_{f}$ et $C_{f}$ sont identifiés sur les courbes de compression statique et font l'objet de l'analyse qui sera présentée ensuite.

\subsection{Procédures de compactage statique}

Les sols testés sont essentiellement des sols fins déstructurés. Quelques-uns résultent de l'écrêtage préalable d'un sol grossier (coupure à 2 ou $5 \mathrm{~mm}$ ). En pratique, le processus de préparation des éprouvettes oedométriques ou triaxiales comprend les étapes suivantes : préparation du sol et traitement éventuel, compactage statique, démoulage et conservation éventuelle. Puis, les éprouvettes sont mises en place dans les cellules d'essai. Elles sont saturées ou pas, selon les objectifs visés, avant d'être prises en charge par la procédure d'essai mécanique.

Une étape préliminaire de préparation des éprouvettes consiste à humidifier le sol pour porter sa teneur en eau

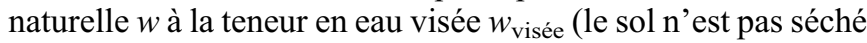
à l'étuve initialement, mais $w<w_{\text {visée }}$ le plus souvent). Cette opération est délicate, car il convient de produire un sol humidifié homogène qui ne forme pas de mottes. Dans ce but, une méthode d'humidification du sol sans malaxage a été proposée par Mieussens (1993). La masse de sol prévue est isolée par quartage. Le sol est étalé sur une plaque portée par une balance, puis il est humidifié par vaporisation d'eau, jusqu'à atteindre la masse humide visée. Le sol est rassemblé en tas à l'aide d'une spatule en évitant de former des mottes, puis il est placé dans un sac transparent étanche, légèrement serré pour réduire au maximum le volume d'air qu'il contient. Le sol foisonné est conservé ainsi pendant 24 heures au moins. Quand le sol est traité, le liant est incorporé au sol foisonné immédiatement avant le compactage statique et il est intégré ainsi dans le processus de densification.

Les éprouvettes triaxiales, dont l'élancement visé est égal à deux, sont préparées dans un moule en acier de $50 \mathrm{~mm}$ de diamètre intérieur et de $250 \mathrm{~mm}$ de hauteur (Fig. 5). La hauteur finale visée est donc de $100 \mathrm{~mm}$. Pour réduire le frottement latéral du sol sur la paroi du moule, le moule est graissé et il est revêtu par une membrane en latex. La membrane est plaquée sur la paroi intérieure du moule par dépression. Le sol foisonné est retiré de son sac de conservation et il est déversé dans le moule sur un piston muni d'une plaque poreuse et d'un évent. La surface du sol est aplanie en évitant toute densification. La 
Tableau 1. Propriétés physiques des sols étudiés.

Table 1. Index properties of the soils studied.

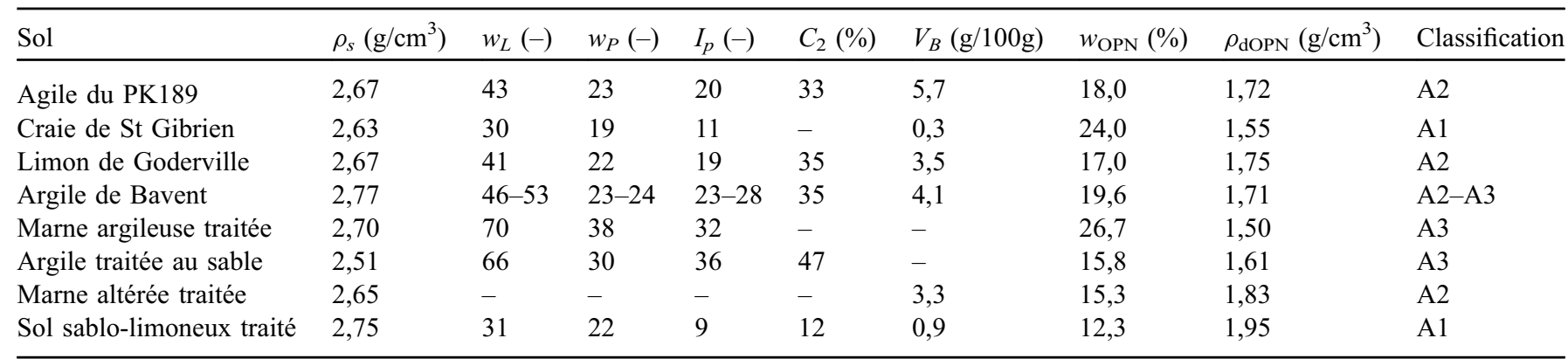

hauteur initiale de l'éprouvette foisonnée dépend de la nature du sol et de sa teneur en eau et se situe autour de $180 \mathrm{~mm}$ généralement. Le sol est coiffé par un piston identique au piston de pied. Le compactage s'effectue entre ces deux pistons flottants à l'aide d'une presse de compression à une vitesse de $5 \mathrm{~mm} / \mathrm{min}$ environ, soit une durée de 20 à 30 minutes. Cette vitesse de tassement correspond à une vitesse de chargement de l'ordre de $50 \mathrm{kPa} / \mathrm{min}$. Cette pratique a été acquise avec l'expérience. Cette vitesse permet d'obtenir un tassement quasi stabilisé à chaque étape du compactage, même si, en toute rigueur, elle devrait être adaptée à la nature du sol. La méthode a pour avantage de produire une éprouvette triaxiale en une seule phase, tout en garantissant une bonne homogénéité du sol compacté (Camapum de Carvalho et al., 1987). Le compactage d'un sol dans un état donné porte sur une éprouvette ou un groupe d'éprouvettes. Trois ou quatre éprouvettes identiques sont compactées pour réaliser un essai triaxial courant, par exemple.

La cellule oedométrique est munie d'une hausse dans laquelle le sol foisonné est déversé. La hauteur initiale de l'éprouvette foisonnée dépend de la nature du sol et de sa teneur en eau et se situe autour de $50 \mathrm{~mm}$ généralement. La compression s'effectue pendant une durée de 20 à 30 minutes, soit une vitesse de chargement de l'ordre de $50 \mathrm{kPa} / \mathrm{min}$, comme ci-dessus. Les éprouvettes sont préparées individuellement ou en groupe. Six éprouvettes identiques sont compactées pour réaliser un essai oedométrique de gonflement-effondrement en parallèle, par exemple.

Le compactage statique s'effectue manuellement à l'aide d'une presse mécanique. La cellule de compactage est placée sur le plateau de la presse et sous un anneau dynamométrique. Le chargement quasi continu s'effectue par petits paliers successifs pendant lesquels sont mesurés l'effort axial $F$ indiqué par l'anneau dynamométrique et le tassement $\Delta h$ qui est mesuré à l'aide d'un réglet. Le chargement est poussé jusqu'à atteindre la hauteur visée de l'éprouvette compactée. Un petit chargement supplémentaire est opéré afin de tenir compte du léger gonflement à attendre pendant le déchargement.

L'effort axial $F$ et le déplacement axial $\Delta h$ mesurés pendant le compactage statique du sol sont convertis en déformation axiale $\varepsilon_{a}=\Delta h / h_{0}$ et en contrainte axiale $\sigma_{a}=F / A$ où $h_{0}$ est la hauteur initiale de l'éprouvette foisonnée et $A$ l'aire de la section du moule de compactage. Le cycle de chargementdéchargement de compactage peut être exprimé au moyen d'une courbe contrainte-déformation $\left(\varepsilon_{a}, \sigma_{a}\right)$ ou, mieux, en fonction de l'indice des vides $e=e_{0}-\left(1+e_{0}\right) \varepsilon_{a}$ où $e_{0}$ est l'indice initial du sol foisonné. L'utilisation de l'indice des vides pour qualifier la compacité permet de mettre en vis à vis l'effet de la teneur en eau et l'effet de la contrainte de compactage (Fig. 4). La courbe de compression du sol foisonné $\left(\log \sigma_{a}, e\right)$ est établie en échelles logarithmiques. Les paramètres $e_{0}, e_{f}, C_{f}$ et $\sigma_{a \max }$ sont identifiés sur la courbe.

L'expérience montre que le compactage statique de plusieurs éprouvettes identiques produit des écarts sur les teneurs en eau de l'ordre de $0,5 \%$ et des écarts sur les masses volumiques de l'ordre de $0,005 \mathrm{~g} / \mathrm{cm}^{3}$. Cela représente des écarts de l'ordre du gramme sur la masse d'une éprouvette triaxiale de $50 \mathrm{~mm}$ diamètre et d'élancement deux. Une connaissance précise de la teneur en eau naturelle du sol avant humidification s'impose donc, afin de ne pas fausser le résultat du compactage par rapport à l'objectif visé. Au total, près de 140 courbes individuelles ou groupes de courbes de compression statique ont été enregistrées ainsi.

\subsection{Nature et propriétés des sols étudiés}

Les données analysées dans cette étude ont été recueillies sur une cinquantaine de sols qui ont été compactés au laboratoire en vue de la réalisation d'essais triaxiaux et oedométriques. Les sols sont déstructurés. Un écrêtage à $2 \mathrm{~mm}$ est opéré sur les sols grossiers (sols graveleux) ou les sols contenant des fragments de grandes dimensions (marnes, craie). Quelques sols sont sableux. Mais, en majorité, les sols testés sont des sols fins limoneux et argileux. Quelques-uns ont été prélevés dans des remblais existants. Les sols ont fait l'objet d'essais d'identification. D'après la classification GTR des sols fins (LCPC et SETRA, 2000), ils se rangent dans la sous-classe A1 (sables fins, limons peu plastiques) et la sousclasse A3 (argiles, argiles marneuses très plastiques) et majoritairement dans la sous-classe A2 (limons, argiles et marnes peu plastiques). Huit de ces sols sont sélectionnés dans la suite pour présenter les courbes de compression statique. Leurs propriétés physiques sont indiquées dans le tableau 1 $\left(C_{2}\right.$, teneur en particules de diamètres inférieurs à $2 \mu \mathrm{m}, V_{B}$ valeur au bleu de méthylène). L'optimum Proctor normal est donné ensuite, puis la classification GTR du sol.

\section{Résultats expérimentaux}

Deux exemples de surfaces d'état sont présentés, qui concernent une argile et une craie déstructurée, puis des 

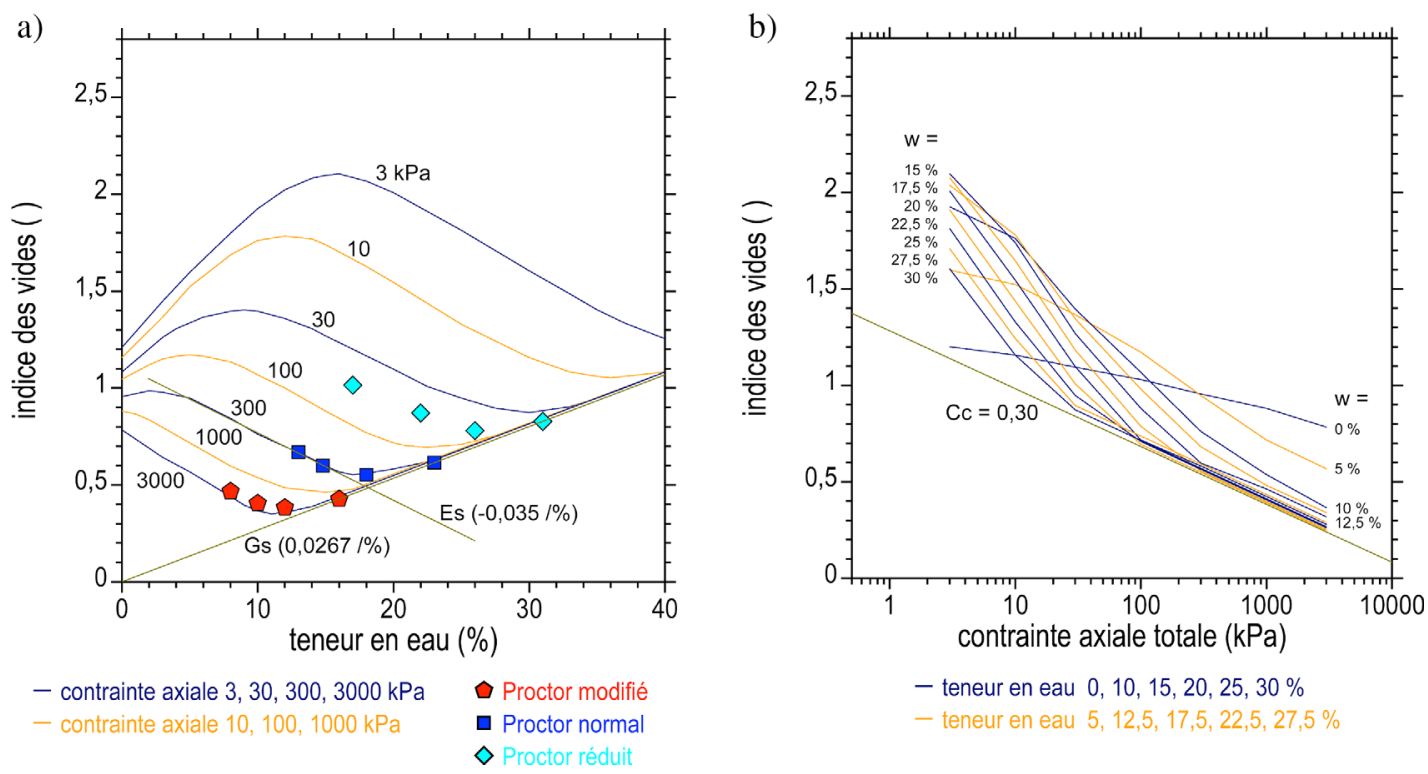

Fig. 6. Surface de compactage statique d'une argile (Serratrice, 1995a) : (a) dans le plan d'état (w,e); (b) dans le plan de compressibilité (log $\left.\left(\sigma_{\mathrm{a}}\right), e\right)$.

Fig. 6. Static compaction surface of a clay: (a) state plane $(w, e)$; (b) compressibility plane $\left(\log \left(\sigma_{a}\right), e\right)$.

courbes de compression statique de sols fins non traités et traités sont commentées ensuite.

\subsection{Surfaces d'état}

\subsubsection{Cas d'une argile}

L'argile a été prélevée dans le flanc d'un remblai autoroutier en vue de procéder à des essais triaxiaux pour mesurer les propriétés de déformabilité et de résistance du sol compacté en différents points d'état (argile du PK189; Serratrice, 1995a). La récapitulation de l'ensemble des courbes de compression statique recueillies pendant la préparation d'une cinquantaine d'éprouvettes permet de construire une surface d'état $\left(w, \log \left(\sigma_{a}\right), e\right)$ qui représente la densification de l'argile à partir des différents états foisonnés. La représentation de cette surface est reproduite sur les graphiques $(w, e)$ et $(\log$ $\left.\left(\sigma_{a}\right), e\right)$ de la figure 6 . La construction d'une telle surface requiert le compactage d'une douzaine d'éprouvettes au moins. La droite de compressibilité de l'argile saturée est obtenue au moyen d'un essai oedométrique effectué sur l'argile malaxée à une teneur en eau de $58 \%$ (1,3 fois sa limite de liquidité), puis mise en place dans l'oedomètre et chargée par paliers de 24 heures. Sa pente est $C_{c}=0,30$. Dans son état foisonné sous $3 \mathrm{kPa}$ par exemple, l'argile possède un minimum de compacité pour une teneur en eau voisine de $16 \%$. Au-delà de cette teneur en eau, le sol ne peut pas être maintenu dans un état lâche. Le sol s'auto-compacte sous l'effet des forces de gravité et des forces capillaires.

Les courbes iso-contraintes axiales déduites des essais sont reportées dans le plan d'état $(w, e)$ de la figure 6a. Elles sont obtenues par interpolation entre les courbes de compression statique de la figure 6b. Les courbes Proctor mesurées sous trois énergies différentes sont reportées dans le plan d'état (essais Proctor réduit, Proctor normal et Proctor modifié réalisés avec des énergies calculées égales à $0,29,0,53$ et
$2,43 \mathrm{MNm} / \mathrm{m}^{3}$ ). Sous les fortes contraintes, les courbes déduites du compactage statique s'apparentent à ces courbes Proctor. Ici, les contraintes axiales équivalentes aux trois énergies Proctor sont respectivement et approximativement égales à 60,300 et $2500 \mathrm{kPa}$.

La figure $6 \mathrm{~b}$ montre les courbes de compression statique dans le plan $\left(\log \left(\sigma_{a}\right), e\right)$. Ces courbes sont des iso-teneurs en eau tant que le degré de saturation reste inférieur à $95 \%$ environ. La forme de ce faisceau s'apparente à celui de la figure 2. Les courbes se situent en-dessus de la courbe de compressibilité de l'argile déstructurée saturée de pente $C_{c}$ et viennent tangenter cette droite à forte pression. Les pentes maximales $C_{f}$ diminuent et passent de 1,5 à 0,55 avec la réduction de la teneur en eau de compactage. Les contraintes axiales qui sont appliquées pour compacter l'argile augmentent rapidement. La poursuite de densification de l'argile par compactage statique ne peut s'effectuer sans expulsion d'eau (consolidation). L'argile devient quasi saturée et les chemins suivis dans le plan d'état $(w, e)$ et le plan $\left(\log \left(\sigma_{a}\right), e\right)$ approchent les droites de pente $C_{c}$ et $G_{s}$. Côté sec, les isocontraintes présentent un maximum, qui s'accorde à la progression des iso-teneurs en eau du plan $\left(\log \left(\sigma_{a}\right), e\right)$ pour les états foisonnés sous les faibles contraintes.

\subsubsection{Cas d'une craie déstructurée}

Un plan d'expériences du même type a été appliqué à une craie déstructurée (craie de St Gibrien; Serratrice, 1995b). La récapitulation de l'ensemble des courbes de compression statique recueillies pendant la préparation d'une centaine d'éprouvettes permet de construire une surface d'état $(w, \log$ $\left(\sigma_{a}\right), e$ ) qui représente la densification de la craie pour des teneurs en eau comprises entre 1 et $36 \%$. La représentation de cette surface est reproduite sur les graphiques $(w, e)$ et $(\log$ $\left(\sigma_{a}\right), e$ ) de la figure 7 . Dans son état foisonné sous $3 \mathrm{kPa}$ par exemple, la craie possède un minimum de compacité pour une 
a)

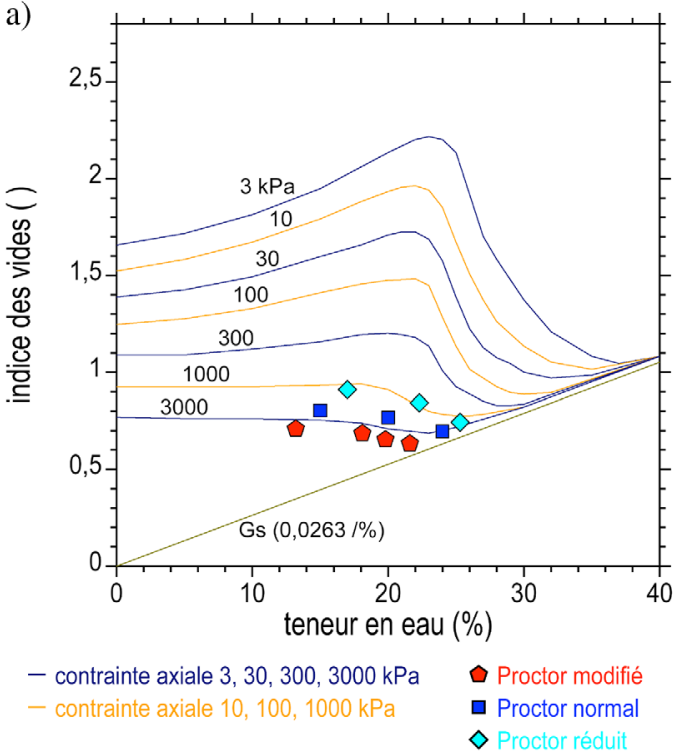

b)

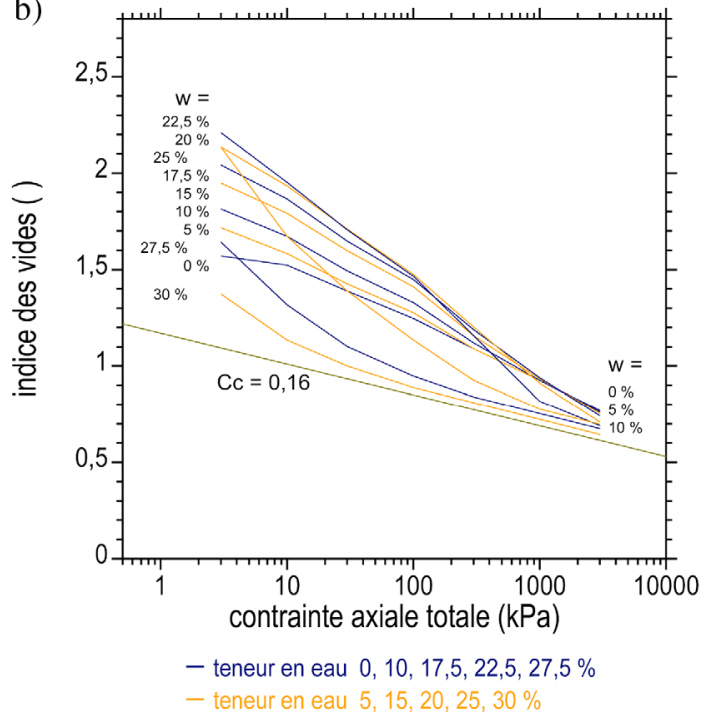

Fig. 7. Surface de compactage statique d'une craie (Serratrice, 1995a): (a) dans le plan d'état (w, e); (b) dans le plan de compressibilité $\left(\log \left(\sigma_{a}\right), e\right)$.

Fig. 7. Static compaction surface of a chalk: (a) state plane (w, e); (b) compressibility plane $\left(\log \left(\sigma_{a}\right), e\right)$.

teneur en eau voisine de $23,5 \%$ dans le plan d'état $(w, e)$. Puis, les courbes iso-contrainte axiale deviennent plus étirées. Un faisceau de courbe de compressibilité se dessine dans le plan $\left(\log \left(\sigma_{a}\right), e\right)$, avec des compressibilités qui dépendent de la teneur en eau (pentes maximales $C_{f}$ comprises entre 0,56 à $1,0)$. Il apparaît ensuite que les contraintes à appliquer pour densifier la craie sont plus fortes que celles mises en œuvre pour densifier l'argile examinée plus haut (Fig. 6). Ainsi, les contraintes axiales équivalentes aux trois énergies Proctor sont respectivement et approximativement égales à 1000, 2500 et $5000 \mathrm{kPa}$ (Proctor réduit, normal et modifié). Les courbes de compression statique (et iso-teneurs en eau) présentent une allure comparable à celles de la figure 2 .

\subsection{Exemples de courbes de compression statique}

Les deux exemples qui suivent sont tirés de plans d'expériences étendus. Mais, dans les applications courantes, les plans d'expériences se réduisent à quelques courbes de compression statique qui ne permettent pas de tracer des lignes isocontraintes axiales dans le plan d'état $(w, e)$. L'équivalence entre le mode de compactage statique et les essais Proctor ou Proctor modifié qui servent de référence ne peut pas être établie précisément. Seule la représentation $\left(\log \left(\sigma_{a}\right), e\right)$ est proposée alors.

\subsubsection{Limon de Goderville}

Le limon de Goderville a servi de support pour l'étude des déformations des sols compactés produites par les variations d'état hydrique (Ferber et al., 2008). Dans ce cadre, un plan d'expériences a été mis en œuvre sur le limon en vue de mesurer ses propriétés mécaniques en différents points d'état, puis examiner la sensibilité de ces déformations aux variations de teneur en eau (Serratrice, 2013). Quelques courbes de compression statique $\left(\log \left(\sigma_{a}\right), e\right)$ sont reportées dans la figure 8 .
Deux essais oedométriques ont été effectués en marge du programme expérimental afin d'obtenir les propriétés de compressibilité du limon saturé. Dans un premier essai oedométrique, le limon est malaxé à une teneur en eau de $58 \%$, donc totalement déstructuré, puis il est mis en place dans une cellule oedométrique et chargé par paliers successifs de 48 heures et plus (un cycle de chargement-déchargement jusqu'à la contrainte maximale $\sigma^{\prime}{ }_{a \mathrm{cmp}}=820 \mathrm{kPa}$ ). La courbe de compressibilité vierge $\left(\log \left(\sigma_{a}\right), e\right)$ ainsi obtenue est représentée dans la figure 8 par des ronds bleus $\left(C_{c}=0,25\right)$. Dans le second essai, le limon déstructuré est placé dans une cellule oedométrique et dans un état foisonné à sa teneur en eau naturelle $(w=5,5 \%, e=1,1)$. Le limon est chargé par paliers jusqu'à une contrainte axiale égale à $100 \mathrm{kPa}$, sous laquelle il est mis en imbibition (triangles verts). L'imbibition provoque un effondrement qui a pour effet de porter le sol dans un état quasi saturé et sur la courbe de compressibilité donnée par l'essai précédent. La suite du cycle de chargement-déchargement est alors identique à celle du limon initialement saturé.

Le graphique $\left(\log \sigma_{a}, e\right)$ de la figure 8 porte trois autres courbes représentées par des étoiles. Il s'agit de courbes de compression statique obtenues à partir de différentes teneurs en eau du limon foisonné $\left(w_{0}=13,8,17,0\right.$ et $\left.19,9 \%\right)$. Trois aspects importants du compactage statique apparaissent ici. Les courbes de compression sont situées au-dessus de la courbe de compressibilité vierge du limon déstructuré saturé. Les pentes maximales $C_{f}$ des courbes de compression augmentent avec l'accroissement des teneurs en eau de compactage $\left(C_{f}=0,82,0,92\right.$ et 0,97$)$. Sous les fortes pressions, les courbes de compression tendent à s'infléchir vers la courbe de compressibilité du limon déstructuré saturé. Il est vraisemblable que des contraintes de compactage de plusieurs dizaines de mégapascals seraient nécessaires pour compacter le limon $\sec \left(w_{0}=5,5 \%\right.$, triangles verts $)$. 


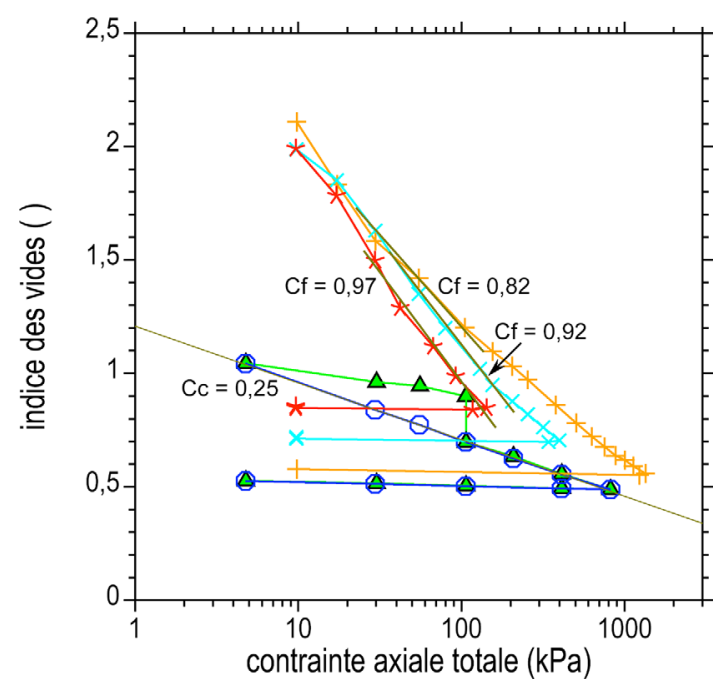

O limon reconstitué saturé $\quad+w=13,8 \% \quad * w=19,9 \%$

$\Delta$ limon reconstitué sec $\quad \times w=17,0 \%$

Fig. 8. Courbes de compression du limon de Goderville dans le plan de compressibilité $\left(\log \left(\sigma_{a}\right), e\right)$.

Fig. 8. Static compression curves of Goderville silt in the compressibility plane $\left(\log \left(\sigma_{a}\right), e\right)$.

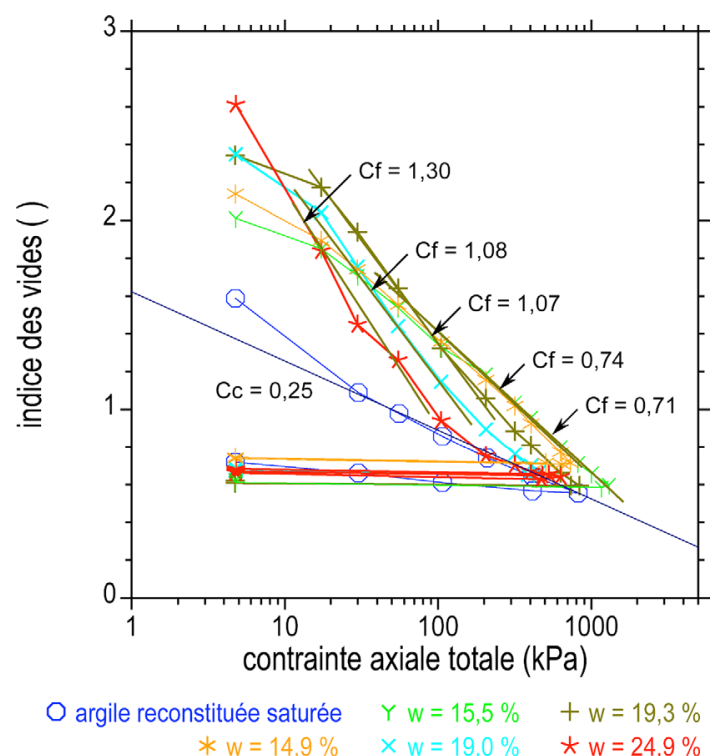

Fig. 9. Courbes de compression statique de l'argile de Bavent dans le plan de compressibilité $\left(\log \left(\sigma_{a}\right), e\right)$.

Fig. 9. Static compression curves of Bavent clay in the compressibility plane $\left(\log \left(\sigma_{a}\right), e\right)$.

\subsubsection{Argile de Bavent}

L'argile de Bavent compactée a fait l'objet d'une expérimentation de transfert hydrique en grandeur (Droniuc, 2013). Les gonflements et les tassements ont été enregistrés à l'aide de tassomètres disposés à différentes profondeurs, durant un cycle d'humidification-séchage de plus de 900 jours. Parallèlement, les propriétés mécaniques de l'argile ont été mesurées au laboratoire sur les éprouvettes compactées par compactage statique. La

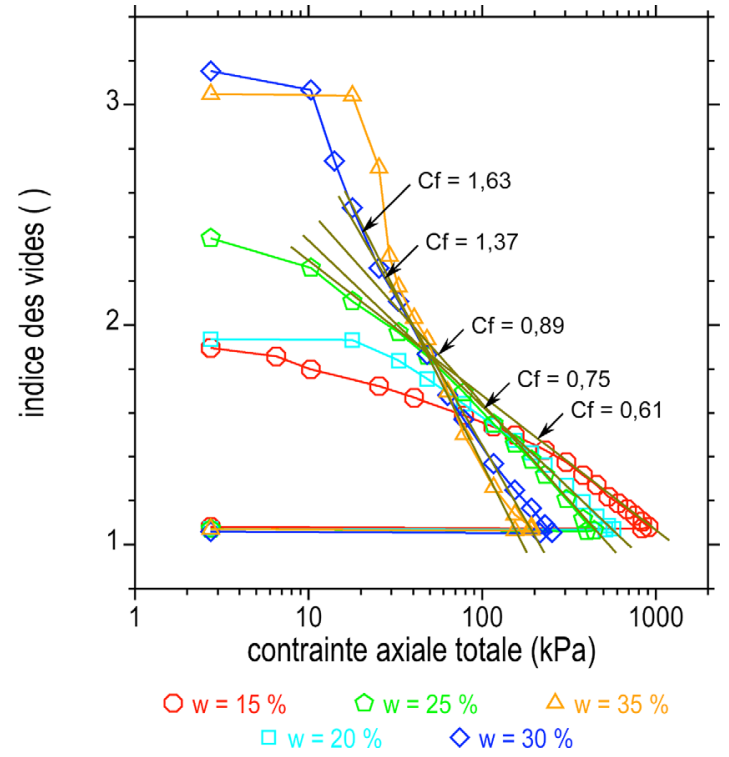

Fig. 10. Courbes de compression statique d'une argile caillouteuse dans le plan de compressibilité $\left(\log \left(\sigma_{a}\right), e\right)$.

Fig. 10. Static compression curves of a clay in the compressibility plane $\left(\log \left(\sigma_{a}\right), e\right)$.

figure 9 montre les courbes de compression oedométrique (log $\left.\left(\sigma_{a}\right), e\right)$ de l'argile en cinq points d'état, comparées à sa courbe de compressibilité vierge de pente $C_{c}=0,25$. Il apparait une translation progressive des courbes vers les contraintes croissantes et un affaissement de leur pente. D'une manière générale, les sols foisonnés plus secs appellent une plus grande énergie de compactage et sont moins compressibles. Les pentes maximales $C_{f}$ vont de 0,7 à 1,3 sur cet exemple.

\subsubsection{Autres sols compactés}

Cinq autres exemples de courbes de compression statique sont présentés ci-dessous. Ces exemples, parmi d'autres, montrent le compactage statique de sols argileux non traités ou traités de différentes façons. Ces données s'inscrivent dans le cadre de projets d'ouvrages. Il n'est donc pas possible d'en fournir tous les détails ici.

Le premier exemple concerne une argile caillouteuse peu plastique qui a été compactée à titre exploratoire. Elle est écrêtée à $2 \mathrm{~mm}$ au préalable et elle s'apparente à une argile de classe A2. Les teneurs en eau visées sont $w_{\text {visée }}=15,20,25,30$ et $35 \%$. Les compacités visées ont été fixées en lien avec l'état $\mathrm{du}$ sol en place à faible profondeur, où il est peu compact. La figure 10 montre cinq courbes de compression statique (log $\left.\left(\sigma_{a}\right), e\right)$ obtenues pour ces différentes teneurs en eau de compactage. La progression des courbes confirme les résultats précédents, avec des pentes maximales $C_{f}$ comprises entre 0,61 et 1,63 et qui augmentent avec la teneur en eau.

\subsection{Exemples concernent des sols traités}

\subsubsection{Marne argileuse traitée}

La figure 11 montre les courbes de compression statique de trois triplets d'éprouvettes triaxiales préparées pour mesurer les propriétés mécaniques du sol traité et du sol non traité. Il 


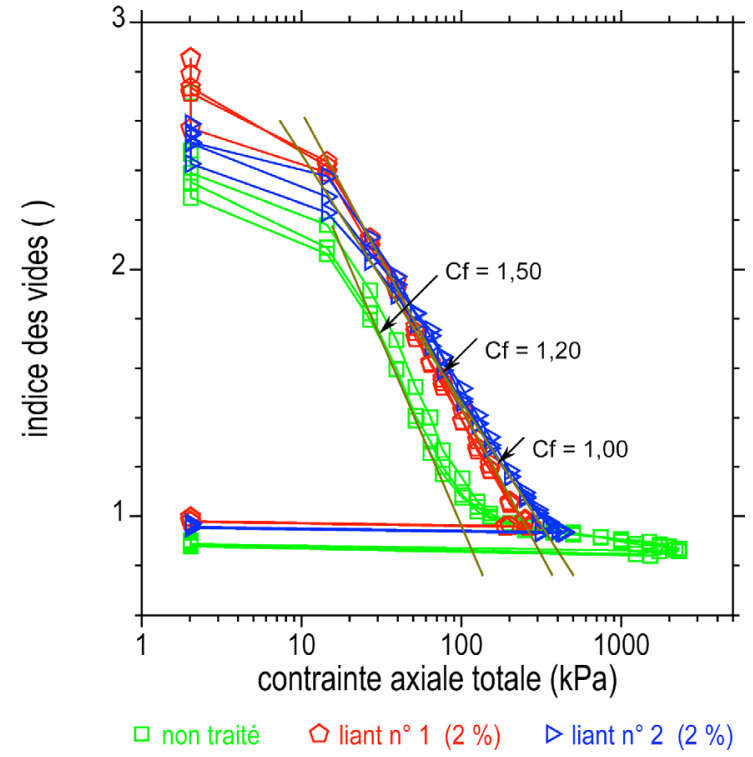

Fig. 11. Courbes de compression statique d'une marne argileuse traitée et non traitée dans le plan de compressibilité $\left(\log \left(\sigma_{a}\right), e\right)$.

Fig. 11. Static compression curves of a clayed marl treated or no in the compressibility plane $\left(\log \left(\sigma_{a}\right), e\right)$.

s'agit d'une marne argileuse déstructurée. Le traitement est effectué au moyen de deux liants différents à la teneur de $2 \%$ (chaux ou liants hydrauliques routiers). Dans cet exemple, le compactage du sol non traité est poussé à haute pression en condition drainée, ce qui explique la forme de la courbe, alors que les chargements des deux sols traités sont interrompus avant ce stade. La présence du liant accroît le foisonnement du sol ici. Puis, les pentes maximales $C_{f}$ des sols traités sont plus faibles que celle du sol non traité.

\subsubsection{Marne altérée traitée}

La figure 12 montre les courbes de compression statique de trois triplets d'éprouvettes triaxiales d'un sol marneux altéré. Le sol est non traité ou il est traité par un liant avec un dosage de 1 et de $2 \%$ (liant hydraulique routier). Les courbes de compression sont données en déformation axiale et en échelles linéaires $\left(\varepsilon_{a}, \sigma_{a}\right)$ ou en indice des vides et en échelles logarithmiques $\left(\log \left(\sigma_{a}\right), e\right)$ dans les figures $12 \mathrm{a}$ et $12 \mathrm{~b}$. Il apparaît clairement que l'énergie à fournir pour compacter le sol traité augmente avec la teneur en liant. Contrairement à la marne argileuse de la figure 11 , le sol non traité foisonné s'avère un peu plus lâche que le sol traité. Mais, comme précédemment, les pentes maximales $C_{f}$ diminuent avec l'accroissement de la teneur en liant. Un effort de compactage statique plus grand doit être appliqué au sol traité pour atteindre un niveau de compacité donné. Inversement, un effort de compactage donné produit une densité du sol traité compacté plus faible que celle du sol non traité. Généralement, le compactage Proctor va dans le même sens.

\subsubsection{Mélange de sable et d'argile}

La figure 13 compare les courbes de compression de deux triplets d'éprouvettes triaxiales d'une argile plastique et le mélange de cette argile avec du sable. Le compactage statique fait référence aux optimums Proctor établis dans chacun des deux cas. La comparaison des courbes de compression de l'argile et du mélange d'argile et de sable confirme les tendances déjà observées pendant le compactage de nombreux sols différents. En état foisonné, les sols argileux sont plus lâches en général que les sols limoneux ou les sols sableux. Les coefficients de compressibilité sont plus grands dans les argiles que dans les sols limoneux et sableux. L'énergie de compactage statique à fournir, qui est représentée par la contrainte axiale ici, est plus grande dans les sols sableux. La contrainte maximale statique à appliquer est plus grande dans les sols frottants. Dans ce sens, des contraintes de plus en plus fortes sont à mettre en œuvre pour compacter les mélanges de sable et d'argile quand la proportion de sable augmente (Moussaï et al., 1993). Sur un plan pratique, les courbes des figures 12 et 13 ont été enregistrées pendant le compactage de plusieurs triplets d'éprouvettes triaxiales. Ces courbes donnent une idée de la bonne répétabilité de la méthode.

\subsubsection{Sol sablo-limoneux traité}

La figure 14 montre les courbes de compression statique de quatre éprouvettes oedométriques. Le sol sablo-limoneux est traité à la bentonite dans les proportions de $0,5,7$ et $12 \%$ d'argile. Le compactage statique fait référence aux optimums Proctor établis dans chacun des cas et qui indiquent un accroissement de $w_{\text {OPN }}$ et une réduction de $\rho_{\text {dOPN }}$ avec la teneur en argile. Le début des courbes de compression statique indique clairement un accroissement de l'indice des vides du sol foisonné $e_{0}$ et de la pente maximale $C_{f}$ avec la teneur en bentonite et la teneur en eau associée $\left(C_{f}\right.$ va de 0,61 à 1,03$)$. Mais, avec des compressibilités de plus en plus faibles quand la charge axiale progresse, les courbes de compression montrent la difficulté de poursuivre la densification du sol, quelle que soit la teneur en argile.

\subsection{5 État du sol foisonné}

Les indices des vides $e_{f}$ sont identifiés avec les pentes $C_{f}$ sur les courbes de compression statique dans le plan $\left(\log \left(\sigma_{a}\right), e\right)$ (Fig. 4b). Dépourvus de signification physique, les indices des vides $e_{f}$ indiquent l'origine des droites de compressibilité sous la contrainte axiale unité $\sigma_{a 0}=1 \mathrm{kPa}$, d'après l'équation (3). Toutefois, ils représentent mal la compacité des sols foisonnés, car les courbes de compression statique montrent souvent un petit palier pseudo-élastique plus dense qui est lié à l'autocompactage initial du sol lors de sa mise en place dans le moule (Fig. 12 et 13 par exemple). Aussi, une représentation plus réaliste de la compacité initiale des sols foisonnés, proche de l'indice des vides initial mesuré $e_{0}$, est donnée par l'indice des vides $e_{10}$ sous $10 \mathrm{kPa}$ de charge axiale et qui vaut $e_{10}=e_{f}-C_{f}$. Ces mesures restent néanmoins très approximatives et dépendent de la méthode de préparation du sol et de sa mise en place dans le moule de compactage. Kodikara (2012) considère cette charge de $10 \mathrm{kPa}$ comme représentative de l'état le plus lâche du sol foisonné après humidification.

\section{Synthèse des résultats et discussion}

\subsection{Contraintes de compactage statique}

Le premier paramètre tiré de l'enregistrement des courbes de compression statique est la contrainte maximale de 
a)

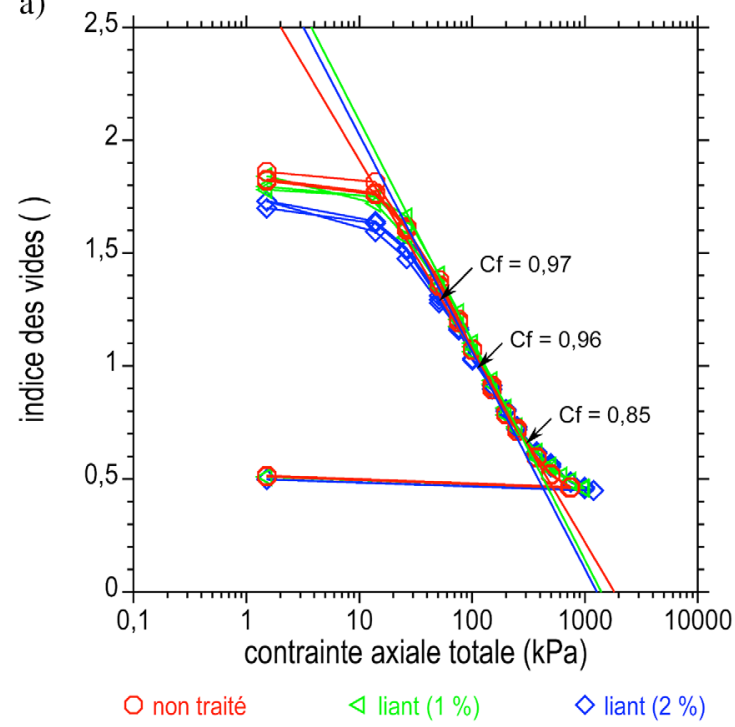

b)

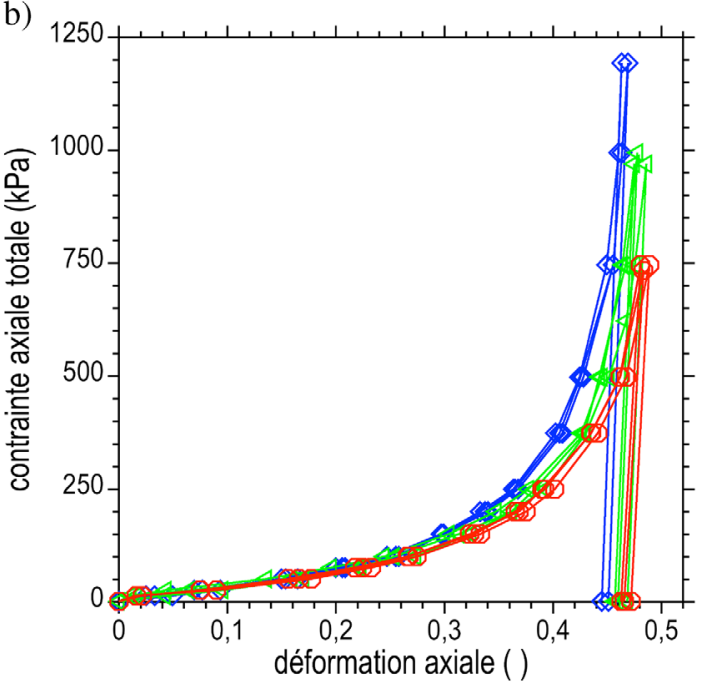

O non traité $\quad \triangleleft \operatorname{liant}(1 \%) \quad \diamond \operatorname{liant}(2 \%)$

Fig. 12. Courbes de compression statique d'une marne altérée traitée et non traitée : (a) dans le plan de compressibilité (log $\left.\left(\sigma_{a}\right), e\right)$; (b) courbes contrainte-déformation dans le plan $\left(\varepsilon_{a}, \sigma_{a}\right)$.

Fig. 12. Static compression curves of a weathereded marl, treated or no: (a) in the compressibility plane (log $\left.\left(\sigma_{a}\right), e\right)$; (b) stress-strain curves $\left(\varepsilon_{a}, \sigma_{a}\right)$.

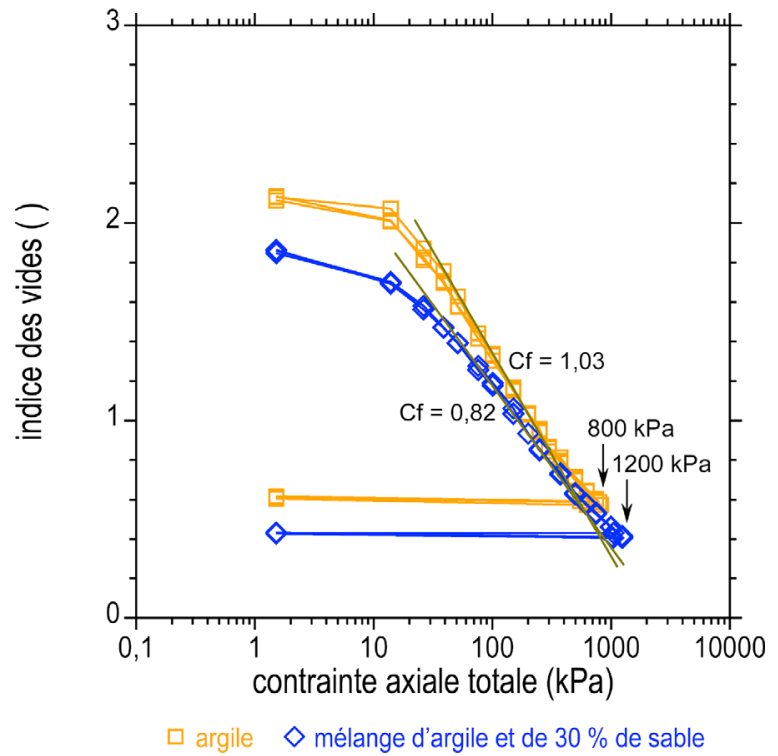

Fig. 13. Courbes de compression statique d'une argile plastique et de l'argile additionnée de sable dans le plan de compressibilité (log $\left.\left(\sigma_{a}\right), e\right)$.

Fig. 13. Static compression curves of a plastic clay and the clay with added sand in the compressibility plane $\left(\log \left(\sigma_{a}\right), e\right)$.

compactage à teneur en eau constante $\sigma_{a c m p}$ (Fig. 4b). Elle s'apparente à une pression de préconsolidation du sol compacté à laquelle il pourra être fait référence ensuite quand les propriétés mécaniques du sol compacté auront été mesurées (pression de préconsolidation effective, contrainte de reprise de compactage, pression de gonflement et seuil d'effondrement sous imbibition, etc.). Quand le degré de saturation du sol compacté reste inférieur au niveau de saturation de la ligne des

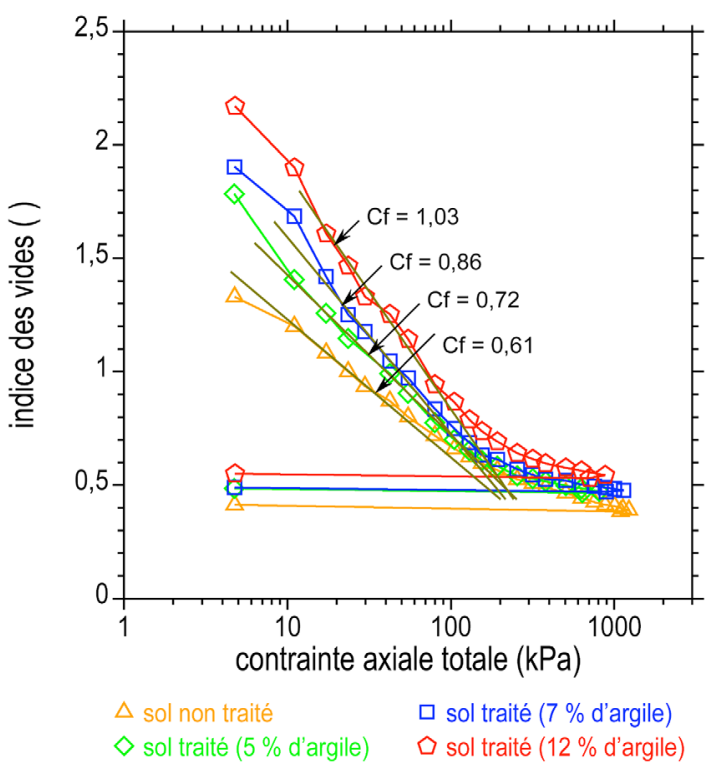

Fig. 14. Courbes de compression statique d'un sol sablo-limoneux traitée à la bentonite dans le plan de compressibilité $\left(\log \left(\sigma_{a}\right), e\right)$.

Fig. 14. Static compaction curves of a sandy silt treated with bentonite clay in the compressibility plane $\left(\log \left(\sigma_{a}\right), e\right)$.

optimums Proctor et son voisinage, vers 90 à $95 \%$ au maximum, la contrainte de compactage peut être reliée aux caractéristiques d'état du sol compacté. Les colonnes 2 à 5 du tableau 2 récapitulent les caractéristiques d'état $w, e$ et $S_{r}$ mesurées après le compactage et les contraintes $\sigma_{\text {acmp }}$ pour atteindre ces états dans les sols compactés non traités dont les courbes de compression statique ont été représentées en partie dans la figure 6 (argile du PK189), la figure 8 (limon de 

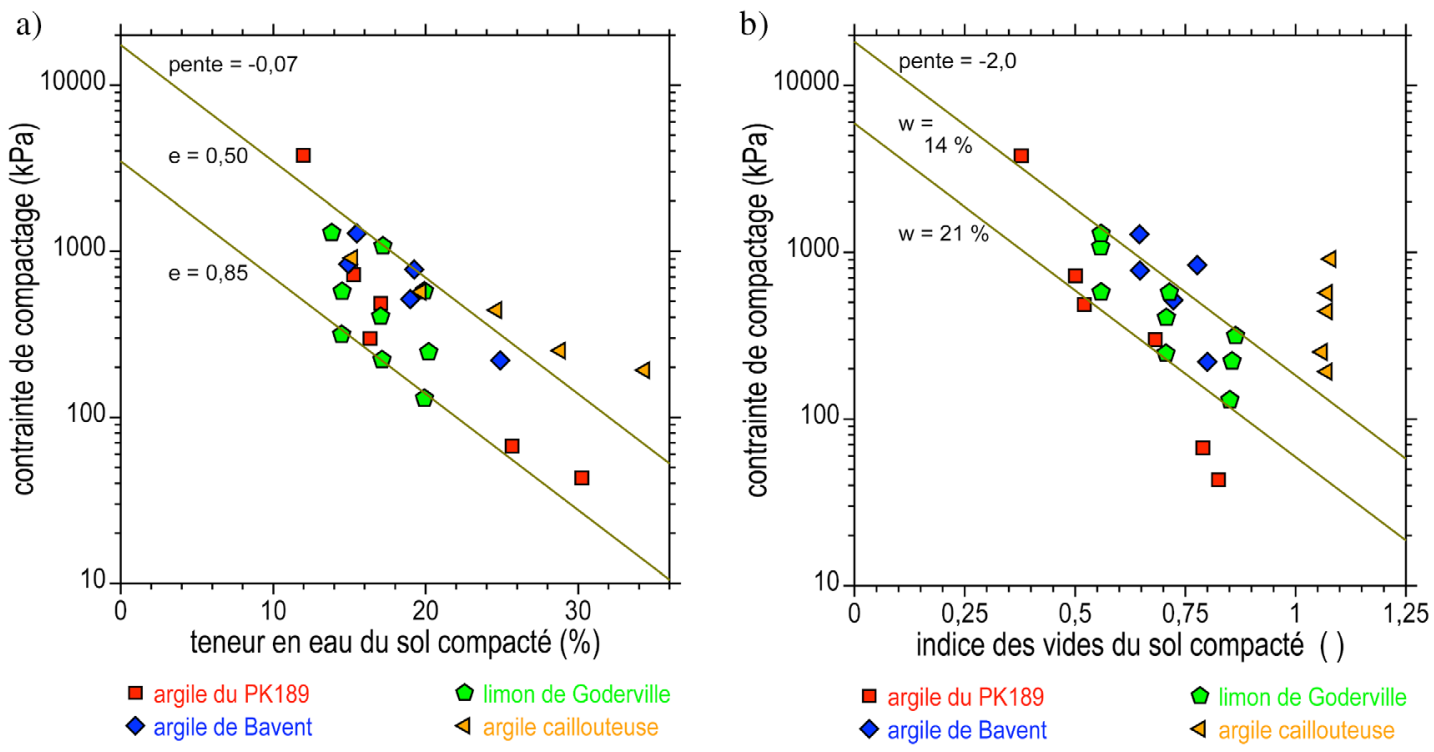

Fig. 15. Contrainte de compactage statique : (a) en fonction de la teneur en eau; (b) en fonction de l'indice des vides du sol compacté.

Fig. 15. Static compaction stress (a) versus water content; (b) versus void ratio of compacted soil.

Goderville), la figure 9 (argile de Bavent) et la figure 10 (argile caillouteuse).

Les contraintes $\sigma_{a c m p}$ sont reportées sur les graphiques de la figure 15 en fonction des caractéristiques d'état $w$ et $e$ des sols compactés. Comme attendu, la contrainte de compactage diminue quand la teneur en eau visée et l'indice des vides visé augmentent. Des encadrements par des droites indiquent cette évolution pour le limon de Goderville (pentagones), avec des pentes égales à $-0,07(\%$ et $\mathrm{kPa})$ et -2 , et avec des ordonnées à l'origine égales à 3,5 et $17,4 \mathrm{MPa}(0,50<e<0,85)$, puis 5,9 et $18,2 \mathrm{MPa}(14<\mathrm{w}<21 \%)$. Sur cette base, une expression de la contrainte de compactage est donnée par:

$$
\log \left(\sigma_{a c m p}\right)=\log \left(\sigma_{a c m p 0}\right)-a w-b e,
$$

où $\sigma_{a c m p 0}, a$ et $b$ sont des constantes qui dépendent de la nature $\mathrm{du}$ sol. Ici, les paramètres $\sigma_{a c m p 0}=174 \mathrm{MPa}, a=0,07$ et $b=2$ représentent le compactage du limon de Goderville dans les conditions du plan d'expériences consacré à ce sol ( $w$ en $\%$ et $\sigma_{a c m p}$ en $\mathrm{kPa}$ ). Les contraintes de compactage calculées $\sigma_{\text {acal }}$ par cette relation à partir des caractéristiques d'état $w$ et $e$ du sol compacté sont reportées dans le tableau 2. Elles sont représentées dans la figure 16 en fonction des contraintes mesurées en échelles bi-logarithmiques. En variant sur deux ordres de grandeur, elles se placent effectivement au voisinage de la bissectrice du graphique.

L'indice des vides $e_{s}$ peut être défini pour représenter chacun des points d'état $(w, e)$ du sol compacté, d'après la relation:

$$
e_{s}=e+E_{s} w
$$

où $E_{s}=a / b$. Dans le plan $(w, e)$, l'indice des vides $e_{s}$ représente l'ordonnée à l'origine des tangentes aux branches des courbes de compactage du côté sec de l'optimum, comme le montre l'exemple de la figure $6 \mathrm{a}$ où $E_{s}=0,035 / \%$. Il est lié à la contrainte et à l'énergie de compactage du sol. Le passage du couple $(w, e)$ à $e_{s}$ s'effectue comme indiqué dans la figure $4 \mathrm{a}$ ( $e=e_{0}$ dans ce cas particulier). Associé au degré de saturation, ils forment un couple de variables indépendantes $\left(e_{s}, S_{r}\right)$ équivalent au couple $(w, e)$ pour caractériser un état du sol compacté. La relation (4) s'écrit alors en fonction de $e_{s}$ (les contraintes sont en $\mathrm{kPa})$ :

$$
\log \left(\sigma_{a c m p}\right)=\log \left(\sigma_{a c m p 0}\right)-b e_{s}
$$

Les paramètres $\sigma_{a c m p 0}, a$ et $b$ ont été identifiés pour les trois autres sols compactés étudiés ci-dessus. Ils sont reportés dans le tableau 3. La pente $E_{s}$ se déduit de $a$ et $b$. Les indices des vides $e_{s}$ sont calculées avec l'équation (5) et sont reportés dans le tableau 2. Les contraintes $\sigma_{\text {acal }}$ calculées avec l'équation (6) sont reportées ensuite. Elles sont représentées dans la figure 16 en fonction de la mesure $\sigma_{a c m p}$. Elles se placent autour de la bissectrice.

Il est intéressant de remarquer que les pentes $E_{S}$ sont égales à 3,5 environ en valeur absolue, pour cet ensemble de quatre sols argileux. Cette pente $E_{s}=3,5$ correspond aussi à la pente de la partie linéaire de la branche côté sec de la courbe Proctor de l'argile du PK189 dans la figure 6a (-0,035/\%), qui représente approximativement la courbe d'iso contrainte de compactage $\sigma_{a}=300 \mathrm{kPa}$ (carrés). En pratique, cette pente n'est pas fixe (ces droites ne sont pas parallèles, voir par exemple Al-Badran et Schanz, 2014). Elle dépend de la nature du sol et du niveau de compactage. Néanmoins, cette valeur $E_{s}=3,5$ donne un ordre de grandeur des pentes des courbes de compactage des sols fins limoneux et argileux, côté sec, c'està-dire des sols compactés à des degrés de saturation compris entre 60 et $90 \%$ environ. L'indice des vides diminue quand la contrainte de compactage augmente. De ce fait, en un point d'état donné $(w, e)$ du sol compacté, l'indice des vides $e_{s}$ est attaché à une contrainte de compactage statique et plus généralement à une énergie de compactage. Il n'est donc pas surprenant de voir l'indice des vides $e_{s}$ jouer le rôle d'une 
Tableau 2. Paramètres tirés des courbes de compression statique.

Table 2. Parameters derived from static compression curves.

\begin{tabular}{|c|c|c|c|c|c|c|c|c|}
\hline Sol & $w(\%)$ & $e(-)$ & $S_{r}(\%)$ & $\sigma_{a c m p}(\mathrm{kPa})$ & $C_{f}(-)$ & $e_{f}(-)$ & $e_{s}(-)$ & $\sigma_{\text {acal }}(\mathrm{kPa})$ \\
\hline \multirow{4}{*}{1} & 25,7 & 0,79 & 87 & 67 & 1,33 & 3,30 & 1,70 & 66 \\
\hline & 17,1 & 0,52 & 87 & 486 & 0,91 & 2,80 & 1,12 & 618 \\
\hline & 15,3 & 0,50 & 81 & 722 & 0,79 & 2,60 & 1,04 & 854 \\
\hline & 12,0 & 0,38 & 85 & 3767 & 0,57 & 2,26 & 0,80 & 2184 \\
\hline \multirow{5}{*}{2} & 14,5 & 0,86 & 45 & 315 & 0,80 & 2,78 & 1,37 & 316 \\
\hline & 20,2 & 0,71 & 77 & 247 & 0,97 & 2,90 & 1,41 & 261 \\
\hline & 14,5 & 0,71 & 55 & 573 & 0,80 & 2,78 & 1,22 & 628 \\
\hline & 17,1 & 0,71 & 65 & 405 & 0,92 & 2,90 & 1,30 & 431 \\
\hline & 19,9 & 0,85 & 63 & 130 & 0,97 & 2,90 & 1,55 & 140 \\
\hline \multirow{3}{*}{3} & 24,9 & 0,80 & 84 & 220 & 1,30 & 3,51 & 1,67 & 144 \\
\hline & 15,5 & 0,65 & 66 & 1281 & 0,74 & 2,90 & 1,19 & 1330 \\
\hline & 14,9 & 0,78 & 53 & 837 & 0,70 & 2,76 & 1,30 & 797 \\
\hline \multirow[t]{5}{*}{4} & 15,2 & 1,08 & 38 & 911 & 0,62 & 2,91 & 1,62 & 1067 \\
\hline & 19,7 & 1,07 & 50 & 570 & 0,75 & 3,13 & 1,78 & 652 \\
\hline & 24,7 & 1,07 & 62 & 442 & 0,89 & 3,43 & 1,95 & 369 \\
\hline & 28,8 & 1,06 & 74 & 252 & 1,37 & 4,19 & 2,09 & 237 \\
\hline & 34,4 & 1,07 & 87 & 192 & 1,63 & 4,65 & 2,30 & 121 \\
\hline
\end{tabular}

variable explicative de la contrainte de compactage dans les exemples présentés ici.

\subsection{Compressibilités des sols foisonnés}

Les tangentes de pentes maximales $C_{f}$ ont été choisies pour caractériser les courbes de compression statique $\left(\log \left(\sigma_{a}\right), e\right)$ des sols foisonnés (Éq. (3)). Recueillies sur près de 140 courbes de compression statique, ces pentes maximales sont reportées en fonction de la teneur en eau de compactage $w_{0}$ sur les graphiques de la figure 17. Chaque croix représente le compactage d'une éprouvette indépendante ou de trois ou six éprouvettes. La dispersion est grande. Mais il n'apparaît pas un moyen simple de normaliser ces données comme avec l'indice des vides $e_{s}$ pour la contrainte de compactage, d'après la relation (6). Un faisceau de droites permet d'encadrer une majorité de réponses $\left(w_{0}\right.$ en $\left.\%\right)$ :

$$
C_{f}=0,05 w_{0}-0,125 \pm 0,375 .
$$

Les réponses particulières des sols examinés plus haut sont reportées sur les graphiques. La figure 17a montre les sols fins compactés à partir de différentes teneurs en eau initiales (argile du PK189, craie de St Gibrien, limon de Goderville, argile de Bavent, argile caillouteuse, dont les courbe de compression statique ont données sur les Fig. 6-10). Les paramètres $e_{f}$ et $C_{f}$ sont reportés dans le tableau 2. La figure $17 \mathrm{~b}$ montre les réponses des sols traités (marne argileuse traitée, marne altérée

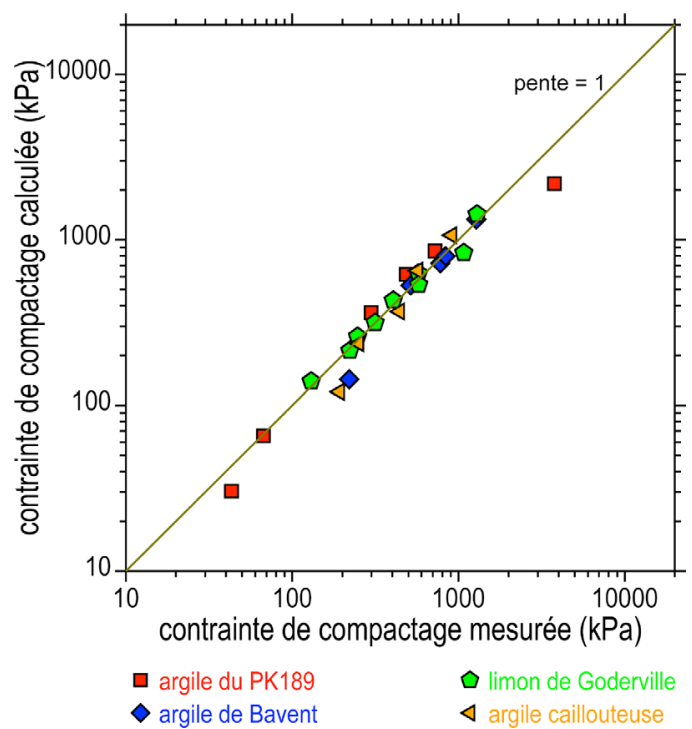

Fig. 16. Contrainte de compactage statique calculée comparée à la contrainte mesurée.

Fig. 16. Static compaction stress calculated compared to the measured stress.

traitée, argile traitée au sable, sol sablo-limoneux traité à la bentonite, dont les courbes sont données dans les Fig. 11-14). Un « $0 »$ indique la réponse du sol non traité. Les autres points donnent la progression des paramètres avec l'accroissement de 
Tableau 3. Paramètres pour les contraintes de compactage statique.

Table 3. Parameters for static compaction stess.

\begin{tabular}{lllcll}
\hline Sol & Origine & $\log \left(\sigma_{a c m p 0}\right)(\mathrm{kPa})$ & $\sigma_{a c m p 0}(\mathrm{MPa})$ & $a(-)$ & $b(-)$ \\
\hline 1 & Argile du PK189 & 4,7 & 50 & 6 & 1,7 \\
2 & Limon de Goderville & 5,5 & 316 & 7 & 2,0 \\
3 & Argile de Bavent & 5,2 & 174 & 7 & 3,53 \\
4 & Argile caillouteuse & 5,3 & 200 & 5 & 2,0 \\
\hline
\end{tabular}

a)

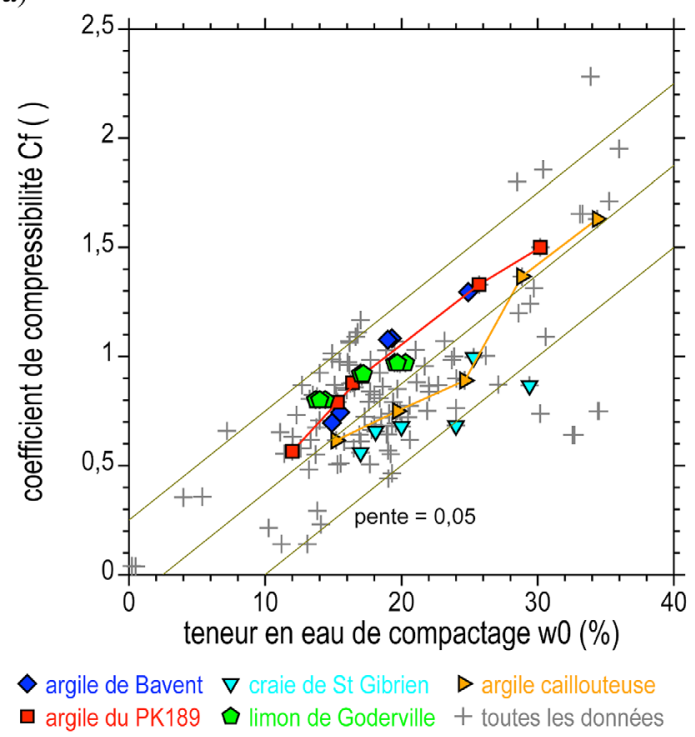

b)

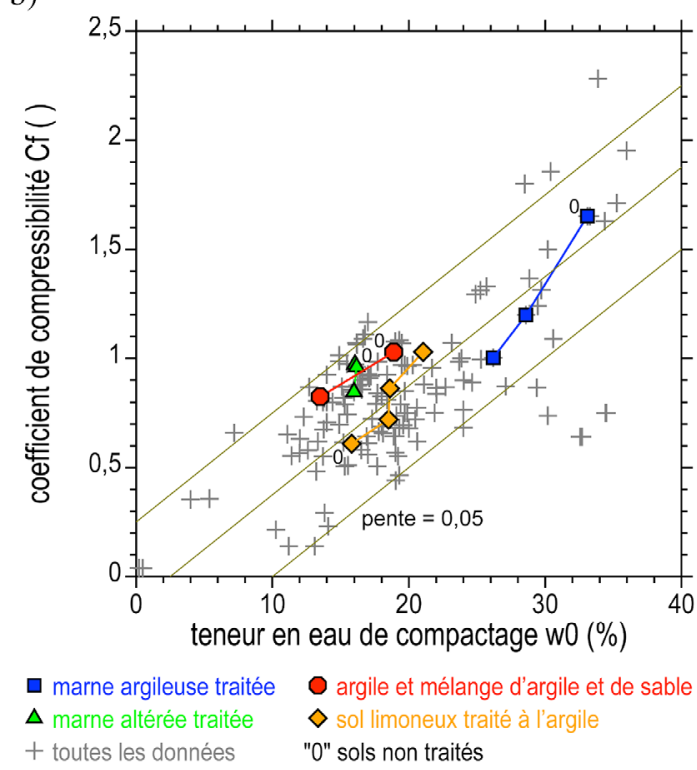

Fig. 17. Caractéristiques des courbes de compression statique. Pentes $C_{f}$ reportées en fonction de la teneur en eau de compactage $w_{0}$. Ensemble des données et illustrations particulières : (a) sols compactés à différentes teneurs en eau; (b) sols traités.

Fig. 17. Characteristics of static compression curves. Slopes $C_{f}$ versus $e_{0}$. All data and illustrations: (a) soils compacted with different water content; (b) treated soils.

la teneur en liant. L'ajout de sable (ronds) produit un effet opposé à l'ajout d'argile (losanges).

Les pentes maximales $C_{f}$ sont représentées en fonction des indices des vides $e_{f}$ sur les graphiques de la figure 18 , où les données particulières sont pointées aussi. Un faisceau de droites permet d'encadrer une majorité de réponses:

$$
C_{f}=0,5 e_{f}-0,60 \pm 0,30 .
$$

À l'intérieur de ce faisceau les données sont triées en trois classes de teneurs en eau initiales $\left(w_{0}<16 \% ; 16 \%<w_{0}\right.$ $<20 \% ; 20 \%<w_{0}$ ) pour confirmer la progression des couples $\left(e_{f}, C_{f}\right)$ avec $w_{0}$.

À l'exception de quelques points proches de l'origine du plan $\left(e_{f}, C_{f}\right)$, qui concernent des sols sableux, les indices des vides $e_{f}$ des sols foisonnés varient entre 1 et 5 et les pentes maximales $C_{f}$ varient entre 0,1 et 2 . À l'état foisonné, les sols secs, qui sont aussi les plus sableux, sont compacts et peu compressibles. À l'opposé, les sols les plus humides foisonnés, qui sont aussi les plus argileux, sont lâches et compressibles. Un tri effectué sur la limite de liquidité ou l'indice de plasticité montre la même tendance. Mais il ne s'agit que de tendances quand l'ensemble des données est pris dans sa totalité. Les cas particuliers pris individuellement montrent effectivement que les paramètres $e_{f}$ et $C_{f}$ augmentent avec la teneur en eau de compactage et ou avec l'argilosité du sol foisonné. Les sols sableux ou les sols argileux secs se trouvent dans des états foisonnés plus denses à l'origine. Mais ils sont moins compressibles. Le traitement du sol foisonné modifie sa réponse. Le traitement à la chaux ou aux liants hydrauliques routiers a pour effet de réduire $e_{f}$ et $C_{f}$ (carrés et triangles dans la Fig. 18b). L'ajout de sable produit un effet similaire (ronds). Au contraire, le traitement à la bentonite produit une augmentation de $e_{c}$ et $C_{f}$ avec l'accroissement de la teneur en argile avec l'accroissement consécutif de la teneur en eau (losanges).

\subsection{Autres variables explicatives du compactage statique}

Les propriétés mécaniques et hydrauliques du sol compacté dépendent directement du nouvel état acquis par le sol après son compactage statique. Parmi les exemples 
a)

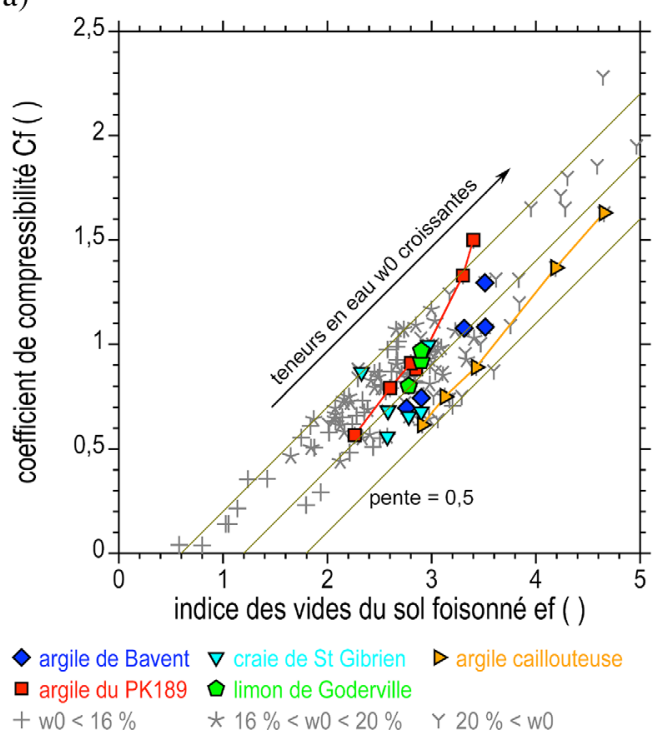

b)

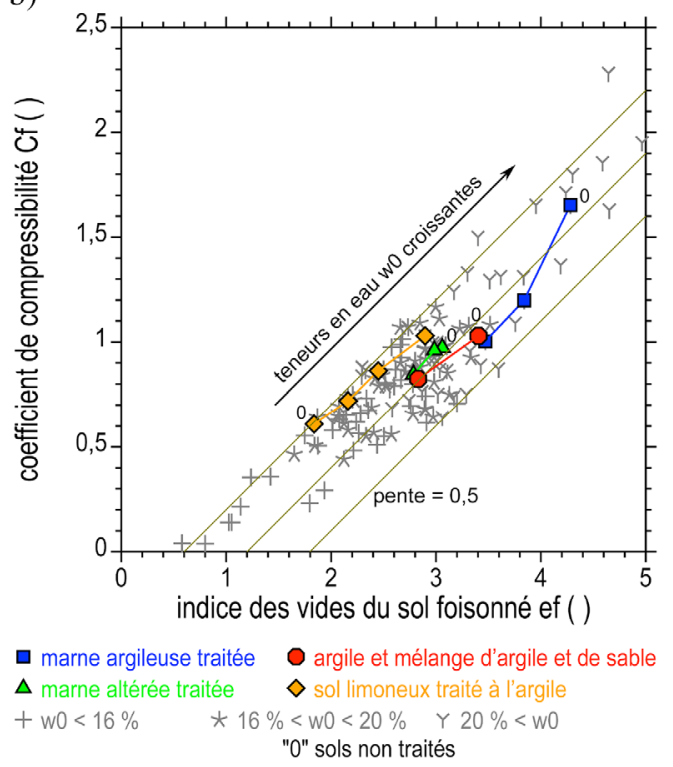

Fig. 18. Caractéristiques des courbes de compression statique. Pentes $C_{f}$ reportées en fonction de $e_{f}$. Ensemble des données et illustrations particulières : (a) sols compactés à différentes teneurs en eau; (b) sols traités.

Fig. 18. Characteristics of static compression curves. Slopes $C_{f}$ versus $e_{f}$. All data and illustrations: (a) soils compacted with different water content; (b) treated soils.

présentés ici, des lois empiriques ont été proposées pour décrire l'évolution de ces propriétés dans le plan d'état $(w, e)$. Tel est le cas de l'argile du PK189, de la craie de St Gibrien et du limon de Goderville (Serratrice, 1995a, b, 2013). Les relations (4) et (6) examinées ci-dessus donnent un exemple d'application à la contrainte de compactage $\sigma_{a c m p}$. Les relations (7) et (8) constituent une autre forme de ce type de relations empiriques. Elles permettent de donner une représentation simplifiée des chemins de compactage (à teneur en eau $w_{0}$ donnée et constante, dans un intervalle donné du degré de saturation $S_{r}$, calcul de $C_{f}$ et $e_{f}$, puis des couples $\left.\left(e, \sigma_{a}\right)\right)$ ou de la surface d'état définie par ses iso- $\sigma_{a}$ dans le plan $(w, e)$, c'est-à-dire des courbes Proctor côté sec (pour la contrainte axiale $\sigma_{a}$ et dans un intervalle donné du degré de saturation $S_{r}$, calcul de $w_{0}, C_{f}, e_{f}$ et $e$, solutions du système des quatre équations linéaires (2), (3), (7) et (8)).

Comme indiqué plus haut (Sect. 2), la mécanique des sols non saturés offre un cadre interprétatif du comportement des sols compactés. Sans donner accès à la mesure ou au contrôle de la succion, l'instrumentation mise en œuvre ici pour enregistrer les courbes de compression statique ne permet pas d'interpréter les réponses observées dans ce cadre. Néanmoins, en pointant le rôle important de la teneur en eau de compactage, l'analyse des données s'inscrit dans la démarche initiée par Kodikara (2012) et reprise ensuite (Abeyrathne et al., 2015 ; Islam et Kodikara, 2016; Kieu et Mahler, 2018) et dans laquelle l'indice des vides $e_{w}$ (proportionnel à la teneur en eau) joue le rôle d'une variable indépendante dans le milieu non saturé. Fondée sur la teneur en eau dont la mesure est aisée, cette démarche vise à établir un lien entre l'approche en milieu non saturé et la pratique.

De même, l'approche microstructurale offre un autre angle de vue pour expliquer le comportement des sols compactés. D'ailleurs, les informations issues de cette approche peuvent être incorporées dans la description du comportement non saturé (Alonso et al., 2013). Ici, en l'absence de ces informations, une analogie est recherchée entre la forme des courbes de compression statique enregistrées et les courbes de compressibilité observées sur d'autres sols, comme ceux évoqués dans la section 2 (voir les Fig. 1-3). L'allure de ces courbes se décline en trois étapes qui sont décrites par Hong et al. (2012) et d'autres, en régime pseudo-élastique, régime de transition et régime post-transition. Dans ces études, les différents auteurs ont fait appel à des analyses microstructurales pour expliquer les effets de structures qui caractérisent les comportements observés et la dégradation de ces effets avec l'accumulation des déformations du sol. Les très fortes compressibilités mesurées pendant le compactage statique des sols foisonnés dépassent largement les compressibilités des sols naturels non organiques observées habituellement. Ces compressibilités des sols foisonnées s'apparentent à celles des argiles sensibles ou celles des sables uniformes sous de très fortes pressions, à une homothétie près sur l'échelle des pressions. Ces différents sols se caractérisent par leur grande porosité initiale. L'évolution de leur structure s'explique par la rupture des contacts entre les grains et/ou les agrégats, le réarrangement et l'écrasement de ceux-ci, la réduction du volume des pores. La densification met en jeu la résistance de ces éléments et de leurs contacts. Ainsi, le frottement interne dans le sol devrait porter une partie de cette information.

À ce stade, s'il n'est pas possible d'accompagner l'analyse des courbes de compression statique présentées dans cette étude par des informations microstructurales, une piste envisagée consiste à mettre en regard les résistances au cisaillement des sols compactés avec leurs propriétés de compactage. Le compactage statique sert à préparer des éprouvettes triaxiales en vue d'une mesure de la résistance au cisaillement du sol compacté. Après compactage, les 
a)

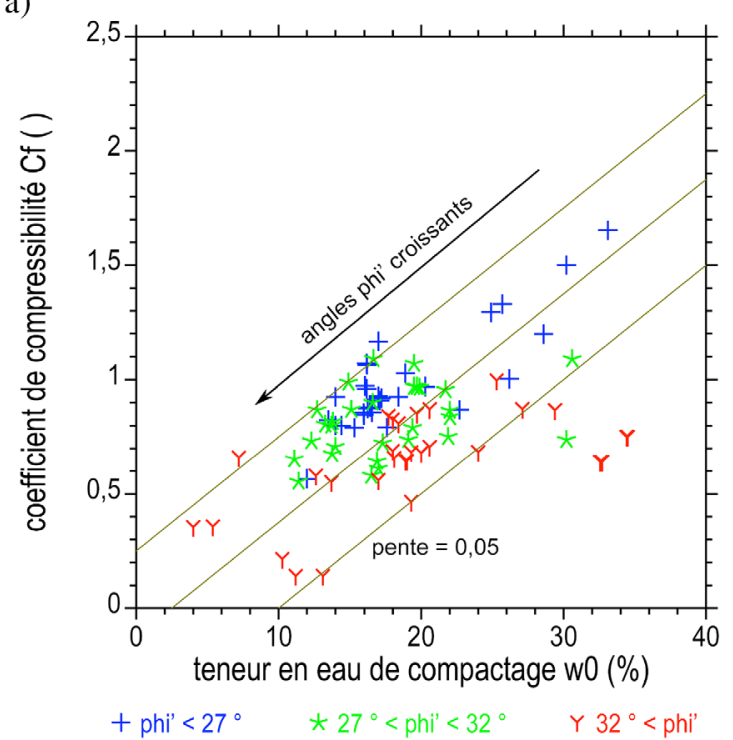

b)

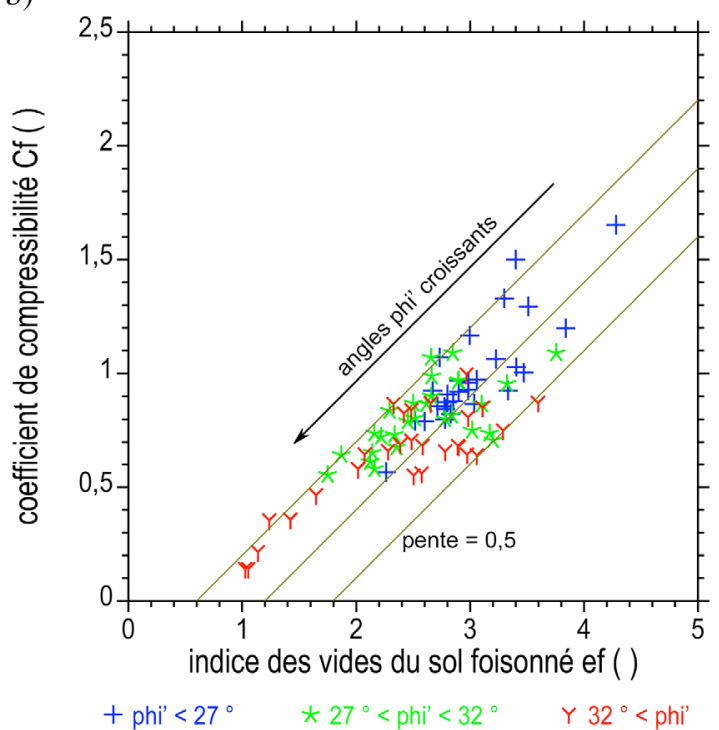

Fig. 19. Lien entre les pentes maximales $C_{f}$ et l'angle de frottement interne: (a) en fonction de la teneur en eau de compactage $w_{0}$; (b) en fonction de $e_{f}$.

Fig. 19. Link between slopes $C_{f}$ and friction ratio: (a) versus compaction water content; (b) versus $e_{f}$.

éprouvettes triaxiales sont saturées sous contre-pression, puis elles sont consolidées et cisaillées. La résistance du sol compacté est caractérisée en contraintes effectives par une cohésion $c$ ' et un angle de frottement interne $\varphi$ ' définie par le critère de Coulomb.

Les pentes maximales $C_{f}$ sont représentées en fonction de la teneur en eau de compactage $w_{0}$ et de l'indice du sol foisonné $e_{f}$ sur les graphiques de la figure 19, avec les encadrements déjà présentés plus haut (Éq. (7) et (8)). Les points représentent le compactage des éprouvettes triaxiales (c'est-à-dire la moitié des données disponibles environ). Ils sont triés en fonction des angles de frottement interne $\varphi$, mesurés après compactage et saturation des sols. Il apparaît ainsi que la pente maximale $C_{f}$ décroît à l'inverse du frottement, comme prévu d'après la nature des sols (argileux, limoneux ou sableux) ou leur état (sols secs). Dans la figure $19 \mathrm{a}$, les points situés à l'écart du fuseau des droites, vers 30 à $35 \%$ de teneur en eau, sont des résidus industriels qui se singularisent par leurs fortes teneurs en eau, leur forte compressibilité et leur très grand frottement interne. Si la résistance au cisaillement du sol compacté saturé ne symbolise qu'indirectement la résistance du sol foisonné pendant le compactage statique, l'angle de frottement interne présente néanmoins un caractère discriminant des paramètres de compactage tirés des courbes analysées ici. Son rôle va à l'opposé de celui de la teneur en eau de compactage. La compacité à l'état foisonné $\left(e_{f}\right.$ ou $\left.e_{10}\right)$ et la compressibilité du sol foisonné sont réduites dans les sols frottants.

\subsection{Apport des courbes de compression statique}

En s'appuyant sur un grand nombre d'exemples, cette étude montre l'intérêt d'enregistrer les courbes de compression statique au laboratoire, car elles font apparaître une grande variété de réponses selon la nature des sols, leur état et leur traitement éventuel. Ces courbes fournissent une contrainte de compactage qu'il est possible de comparer à l'énergie Proctor. Cette contrainte constitue la marque d'une pression de préconsolidation du point de vue de l'histoire du sol compacté. Mais surtout, sur la base d'essais faciles à mettre en œuvre et la mesure de caractéristiques élémentaires (teneur en eau, indice des vides), les courbes de compression statique présentent une allure caractéristique partagée par les sols structurés, qui sont dotés d'une grande porosité et d'une forte compressibilité. L'observation microscopique confirme ces effets de structure et leur dégradation dans les argiles sensibles ou les sables à très haute pression. Par analogie, l'évolution de la structure initiale du sol foisonné vers celle du sol compacté agit sur la forme des courbes de compression statique.

L'enregistrement des courbes de compression statique d'un sol permet de construire une surface d'état, qui est exprimée dans le plan d'état $(w, e)$ et le plan de compressibilité (log $\left(\sigma_{a}\right), e$ ) et qui relie la teneur en eau, l'indice des vides et la contrainte axiale statique. Les propriétés mécaniques et hydrauliques du sol compacté peuvent être attachées à chacun des points de cette surface d'état (comme la résistance au cisaillement par exemple ou des modules de déformation). En références aux courbes de compressibilité des sols structurés, les courbes de compression statique sont caractérisées par deux paramètres, $C_{f}$ et $e_{f}$. Ces paramètres dépendent de la teneur en eau de compactage $w_{0}$, de l'argilosité du sol et ils s'avèrent en lien avec l'angle de frottement interne du sol compacté saturé. Des lois empiriques liant $C_{f}$ et $e_{f}$ à $w_{0}$ indiquent la tendance globale et se vérifient dans chaque cas particulier, y-compris en présence d'un traitement du sol. Ces relations permettent de décrire les chemins de compactage et la surface d'état, en première approximation. Elles donnent ainsi une approximation des courbes Proctor côté sec.

Ainsi, devant la diversité des réponses observées, chaque courbe de compression statique révèle le caractère particulier 
du sol qu'elle représente et de son état. Les courbes de compressibilité statique sont discriminantes dans le processus de compactage des sols au laboratoire. Elles portent une information préliminaire qui semble utile et complémentaire à la caractérisation du comportement mécanique du sol compacté.

\section{Conclusion}

Le compactage statique sert à reconstituer des éprouvettes de sols fins compactés au laboratoire. La densification du sol s'effectue en compression oedométrique monotone. La courbe de compression statique est enregistrée, pour décrire l'évolution de la déformation volumique du sol non saturé. Selon une expression logarithmique classique, elle est caractérisée par une droite tangente qui décrit la forte compressibilité du sol foisonné. Cette expression dépend de deux paramètres, le coefficient de compressibilité $C_{f}$ et l'indice des vides $e_{f}$ du sol foisonné, qui sont identifiés sur les courbes. Ces deux paramètres croissent avec la teneur en eau de compactage. Cette tendance s'avère plus affirmée dans les argiles que dans les sols limoneux ou sableux, si bien que l'argilosité du sol figure aussi parmi les paramètres explicatifs du processus. De même, l'angle de frottement interne du sol compacté saturé possède un pouvoir explicatif des faibles compressibilités des sols foisonnés. Son rôle va à l'opposé de celui de la teneur en eau de compactage.

Ainsi, les courbes de compression statique s'avèrent discriminantes de la nature des sols, argileux, limoneux ou sableux, de la présence de liants et de leur type. Les sols frottants, les sols secs, les sols traités (chaux ou liants hydrauliques routiers) s'avèrent plus résistants au compactage et moins sensibles à la teneur en eau de compactage que les sols argileux. Ils appellent à mettre en œuvre une plus grande énergie de compactage pour atteindre une compacité donnée. Plus généralement, le compactage statique d'un sol peut être décrit par une surface de l'espace $\left(w, e, \log \left(\sigma_{a}\right)\right)$, qui donne une cartographie du processus de densification du sol non saturé sur les chemins monotones. Cette approche globale révèle de nombreux aspects du compactage et des propriétés des sols fins compactés. Une correspondance s'établit entre la contrainte compactage statique et l'énergie Proctor. Les argiles se compactent sous des charges nettement plus faibles que les limons, la craie ou les sols secs. Au plan pratique, cette surface de l'espace $\left(w, e, \log \left(\sigma_{a}\right)\right)$ s'appuie sur des caractéristiques élémentaires d'état du sol. Elle donne accès aux variations volumiques du sol foisonné non saturé sur la base d'essais simples à mettre en œuvre.

Cette première récapitulation appelle à recueillir encore de nouvelles données et affiner leur interprétation, puis à envisager la réalisation de nouveaux plans d'expériences détaillés ou des campagnes systématiques d'essais. Dans cette perspective, il conviendrait de mieux décrire le foisonnement des sols, puis de tenter de visualiser les mécanismes qui entrent en jeu à l'échelle microscopique, comme dans les autres domaines auxquels il a été fait références ici, avec les argiles sensibles, les sables et d'autres sols compactés. La question touche aussi l'effet de la présence des liants dans le processus du compactage statique. Sur un autre plan, en donnant accès au suivi du chemin des contraintes totales pendant la compression statique, l'enregistrement de la contrainte radiale oedométrique apportera des données complémentaires utiles. Enfin, comme le montrent des études récentes, les techniques de mesures en usage dans les sols non saturées devront être mises à profit pour accompagner ces expérimentations et élargir ainsi le cadre interprétatif des données recueillies. Avec ces nouvelles avancées, la méthode de compactage statique devrait trouver sa place dans les programmes expérimentaux appliqués et destinés à faire progresser les connaissances des sols compactés et des sols traités compactés.

\section{Principales notations}

$\begin{array}{ll}C_{c} & \text { Coefficient de compressibilité } \\ C_{f} & \begin{array}{l}\text { Pente maximale des courbes de compression } \\ \text { statique }\end{array} \\ e & \text { Indice des vides } \\ e_{0} & \text { Indice des vides initial du sol foisonné (mesuré) } \\ e_{10} & \text { Indice des vides du sol foisonné (sous } 10 \mathrm{kPa}) \\ e_{f} & \text { Indice des vides associé à la pente } C_{f} \\ e_{s} & \text { Ordonnée à l'origine des tangentes aux branches } \\ & \text { des courbes de compactage du côté sec } \\ E_{s} & \text { Pente des tangentes aux branches des courbes de } \\ & \text { compactage du côté sec } \\ G_{s} & \text { Densité des grains solides } \\ w & \text { Teneur en eau } \\ w_{0} & \text { Teneur en eau de compactage } \\ S_{r} & \text { Degré de saturation } \\ \varepsilon_{a} & \text { Déformation axiale } \\ \sigma_{a} & \text { Contrainte axiale totale } \\ \sigma_{a 0} & \left.\text { Contrainte axiale unité ( } \sigma_{a 0}=1 \mathrm{kPa}\right) \\ \sigma_{a c a l} & \text { Contrainte maximale de compactage calculée } \\ \sigma_{a c m p} & \text { Contrainte maximale de compactage à teneur en } \\ \sigma_{a c m p 0} & \text { eau constante } \\ \sigma_{a \max } & \text { Paramètre } \\ & \text { Contrainte axiale maximale appliquée pendant le } \\ & \text { compactage }\end{array}$

\section{Références}

Abeyrathne A, Islam T, Kodikara J, Bui H. 2015. Application of MPK framework to interpret volume change behavior of compacted unsaturated soils. Proceedings of the 6th Asia-Pacific Conference on Unsaturated Soils, Guilin, China, 23-26 October 2015, pp. 749-752. doi: 10.1201/b19248-125.

AFNOR (Association Française de Normalisation). 2018. Terrassement. Parties 1 à 6.

Al-Badran Y, Schanz T. 2014. Modelling the compaction curve of fine-grained soils. Soils Found 54(3): 426-438.

Alonso EE, Pinyol NM, Gens A. 2013. Compacted soil behaviour: initial state, structure and constitutive modelling. Géotechnique 63 (6): 463-478. doi: 10.1680/geot.11.P.134.

Altuhafi FN, Coop MR. 2011. Changes to particle characteristics associated with the compression of sands. Géotechnique 61(6): 459-471.

Andrei S. 1977. Propriétés hydrauliques et mécaniques des sols non saturés. Rev Fr Géotech 2: 49-78. 
Azzouz AS, Krizek RJ, Corotis RB. 1976. Regression analysis of soil compressibility. Soils Found 16(2): 19-29.

Bal'shin MY. 1938. Metal ceramics. Metallurgizdat (en russe).

Bell JR. 1977. Compaction energy relationship of cohesive soils. Transport Res Rec 641: 29-34.

Biarez J, Hicher P-Y. 1997. Influence de la granulométrie et de son évolution par ruptures de grains sur le comportement mécanique de matériaux granulaires. Rev Fr Génie Civil 1(4): 607-631.

Biarez J, Fleureau JM, Zerhouni MI, Soepandji BS. 1987. Variations de volume des sols argileux lors de cycles de drainagehumidification. Rev Fr Géotech 41: 63-71. doi: 10.1051/ geotech/1987041063.

Bosi F, Piccolroaz A, Gei M, Dal Corso F, Cocquio A, Bigoni D. 2013. Experimental investigation of the elastoplastic response of aluminum silicate spray dried powder during cold compaction. $J$ Eur Ceram Soc. doi: 10.1016/j.jeurceramsoc.2013.11.037.

Burland JB. 1990. On the compressibility and shear strength of natural clays. Géotechnique 40(3): 329-378.

Burton GJ, Sheng D, Airey D. 2014. Experimental study on volumetric behaviour of Maryland clay and the role of degree of saturation. Can Geotech J 51(12): 1449-1455. doi: 10.1139/cgj-2013-0332.

Camapum de Carvalho J, Crispel JJ, Mieussens C, Nardone A. 1987. $\mathrm{La}$ reconstitution des éprouvettes en laboratoire. Théorie et pratique opératoire. Rapport de Recherche des LPC, 145, juin $1987,54 \mathrm{p}$.

Casagrande A. 1932. The structure of clay and its importance in foundation engineering. J Boston Soc Civil Eng 19(4): 168-209.

Cerato AB, Lutenegger A. 2004. Determining intrinsic compressibility of fine-grained soils. J Geotech Geoenviron Eng 130(8): 872-877.

Chandler HW, Sands CM, Song JH, Withers PJ, McDonald SA. 2008. A plasticity model for powder compaction processes incorporating particle deformation and rearrangement. Int J Solids Struct 45: 2056-2076.

Chong SH, Santamarina JC. 2016. Soil compressibility models for a wide stress range. J Geotech Geoenviron Eng. doi: 10.1061/ (ASCE)GT.1943-5606.0001482.

Chuhan FA, Kjeldstad A, Bjørlykke K, Høeg K. 2003. Experimental compression of loose sands: Relevance to porosity reduction during burial in sedimentary basins. Can Geotech J 40: 995-1011.

Cotecchia F, Chandler RJ. 2000. A general framework for the mechanical behaviour of clays. Géotechnique 50(4): 431-447. doi: 10.1680/geot.2000.50.4.431.

Cotecchia F, Cafaro F, Guglielmi S. 2016. Microstructural changes in clays generated by compression explored by means of SEM and Image Processing. Procedia Eng 158: 57-62.

Crispim FA, Lima D, Schaefer CEGR, et al. 2011. The influence of laboratory compaction methods on soil structure: Mechanical and micromorphological analyses. Soils Rocks 34(1): 91-98.

Cui YJ, Delage P. 1996. Yielding and plastic behaviour of an unsaturated compacted silt. Geotechnique 46(2): 291-311.

Cuisinier O, Laloui L. 2004. Fabric evolution during hydromechanical loading of a compacted silt. Int J Numer Anal Methods Geomech 28(6): 483-499.

Dadda A, Ghabezloo S, Feia S, Sulem J. 2015. Fracturation des grains et l'évolution de la microstructure d'un sable sous fortes contraintes. Conference Paper, October 2015. doi: 10.13140/ RG.2.1.1976.2002.

Daouadji A, Hicher PY, Rahma A. 2001. An elastoplastic model for granular materials taking into account grain breakage. Eur JMech -A/ Solids 20(1): 113-137. doi: 10.1016/S0997-7538(00)01130-X.

De Bono JP, McDowell GR. 2017. Validation of the $\log$ e-log $\sigma$ normal compression law using particle strength data. Geotechnique. doi: 10.1680/jgeot.17.T.007
Delage P, Audiguier M, Cui YJ, Howat D. 1996. Microstructure of a compacted silt. Can Geotech J 33(1): 150-158.

Delage P. 2010. A microstructure approach to the sensitivity and compressibility of some Eastern Canada sensitive clays. Géotechnique 60(5): 353-368. doi: 10.1680/geot.2010.60.5.353

Delage P, Fry JJ. 2000. Comportement des sols compactés : apports de la mécanique des sols non saturés. Rev Fr Géotech 92: 17-29.

Doris-Asmani MY, Hafez MA, Nurbaya S. 2011. Static laboratory compaction method. Electron J Geotech Eng 16 M: 1583-1593.

Droniuc N. 2013. Étude par la méthode des éléments finis du comportement des remblais en sols fins compactés. Proceeding of the 18th International Conference on Soil Mechanics and Geotechnical Engineering, Paris, France, pp. 1097-1100.

Ekwue EI, Birch R, Chewitt J. 2014. Effect of dynamic and static methods of compaction on soil strength. The West Indian J Eng 37(2): 74-78.

Ferber V, Auriol JC, Cui YJ, Magnan JP. 2008. Comportement des sols fins compactés à l'humidification. Apport d'un modèle de microstructure. Rev Fr Géotech 122: 13-24.

Fleureau JM, Kheirbek-Saoud S. 1992. Variations de résistance des sols compactés avec la pression interstitielle négative. Rev $\mathrm{Fr}$ Géotech 59: 57-64.

Fredlund DG, Rahardjo H. 1993. Soil mechanics for unsaturated soils. New York: John Wiley \& Sons, Inc.

Golightly CR. 1989. Engineering properties of carbonate sands. Ph.D. dissertation, Bradford University, UK.

Guérif J. 1982. Compactage d'un massif d'agrégats : effet de la teneur en eau et de la pression appliquée. Agronomie, EDP Sciences 2(3): 287-294.

Hafez MA, Doris Asmani MY, Nurbaya S. 2010. Comparison between static and dynamic laboratory compaction methods, Electron J Geotech Eng 15: 1641-1650.

Hagerty MM, Hite DR, Ullrich CR, Hagerty DJ. 1993. Onedimensional high-pressure compression of granular media. $J$ Geotech Eng 119(1): 1-18.

Han LH, Elliott JA, Bentham AC, Mills A, Amidon GE, Hancock BC. 2008. A modified Drucker-Prager Cap model for die compaction simulation of pharmaceutical powders. Int $J$ Solids Struct 45: 3088-3106.

Hattab M, Hammad T, Fleurau JM. 2010. Compressibility behaviour of soft clay sediments. Proceeding of the GeoShanghai Conférence, May 2010, 6 p. doi: 10.1061/41101(374)4.

Hattab M, Hammad T, Fleureau JM, Hicher PY. 2013. Behaviour of a sensitive marine sediment: microstructural investigation. Géotechnique 63(1): 71-84.

Hicher PY, Kim MS, Rahma A. 1995. Experimental evidence and modelling of grain breakage influence on mechanical behaviour of granular media. In: International Workshop, Homogenization, Theory of Migration and Granular Bodies, Gdansk-Kormoran, pp. $125-133$.

Hilf JW. 1956. An investigation of pore water pressures in compacted cohesive soils, Technical Memorandum 654, U. S. Department of the Interior, Bureau of Reclamation, Denver, Colorado.

Hong ZS, Zeng LL, Cui YJ, Cai YQ, Lin C. 2012. Compression behaviour of natural and reconstituted clays. Géotechnique 62(4): 291-301.

Horpibulsuk S, Katkan W, Apichatvullop A. 2008. An approach for assessment of compaction curves of fine-grained soils at various energies using a one point test. Soils Found 48(2): 115-126.

Horpibulsuk S, Suddeepong A, Chamket P, Chinkulkijniwat A. 2013. Compaction behavior of fine-grained soils, lateritic soils and crushed rocks. Soils Found 53(1): 166-172.

Islam T, Kodikara J. 2016. Interpretation of the loading-wetting behaviour of compacted soils within the "MPK" framework. Part I: 
Static compaction. Can Geotech J 53(5): 783-805. doi: 10.1139/ cgj-2014-0316.

Jennings JE, Burland JB. 1962. Limitations to the use of effective stresses in partly saturated soils. Géotechnique 12(2): 125-144.

Joslin JC. 1959. Ohio's typical water-density curves. American Society for Testing and Materials. Special Technical Publication STP239, pp. 111-118.

Juarez-Badillo E. 1981. General compressibility equation for soils. Proceedings of the 10th International Conference on Soil Mechanics and Foundation Engineering, Stockholm, Sweden, pp. 171-178.

Keller T, Lamande M, Peth S, et al. 2013. An interdisciplinary approach towards improved understanding of soil deformation during compaction. Soil Tillage Res 128: 61-80.

Kieu MT, Mahler A. 2018. A study on the relationship between matric suction and the void ratio and moisture content of a compacted unsaturated soil. Period Polytech Civil Eng 62(3): 709-716. doi: 10.3311/PPci.11974.

Kodikara J. 2012. New framework for volumetric constitutive behaviour of compacted unsaturated soils. Can Geotech J 49(11): 1227-1243. doi: 10.1139/t2012-084.

Kodikara J, Islam T, Rajeev P. 2016. Interpretation of the loadingwetting behaviour of compacted soils within the "MPK" framework. Part II: Dynamic compaction. Can Geotech J 53(5): 806-827. doi: 10.1139/cgj-2014-0317.

Koliji A, Laloui L, Vulliet L. 2009. Behaviour of unsaturated aggregated soil in oedometric condition. Soils Found 49(3): 369-380.

Kumar R, Jain PK, Dwivedi P. 2016. Prediction of compression index $\left(\mathrm{C}_{\mathrm{c}}\right)$ of fine grained remolded soils from basic soil properties. Int $J$ Appl Eng Res 11(1): 592-598.

Lambe TW. 1958. The engineering behaviour of compacted clay. $J$ Soil Mech Found Divis, ASCE 84(2): 1-35.

Lambe TW. 1961. Residual pore pressures in compacted clay. Proceeding 5th International Conference on Soil Mechanics and Foundation Engineering, Paris, France, 1/35, pp. 207-211.

Lawton EC, Fragaszy RJ, Hetherington MD. 1992. Review of wetting-induced collapse in compacted soil. J Geotech Eng 118(9): 1376-1394. doi: 10.1061/(ASCE)0733-9410(1992)118:9(1376)

LCPC, SETRA. 2000. Guide technique pour les terrassements routiers (GTR). Réalisation des remblais et des couches de forme. Juillet 2000, $2^{\mathrm{e}}$ Édition. Fascicule I, Principes généraux, 98 p, Fascicule II, Annexes techniques, $102 \mathrm{p}$.

Leclercq J, Verbrugge JC. 1985. Propriétés géomécaniques des sols non saturés. Compte rendu du Colloque Int. sur le Travail du Sol, Faculté des Sciences Agronomiques de Gembloux, Gembloux, Belgique, pp. 1-8.

Leonards GA. 1955. Strength characteristics of compacted clays. Trans ASCE 120: 1420-1454.

Leong EC, Widiastuti S, Rahardjo H. 2013. Estimating wettinginduced settlement of compacted soils using oedometer test. Geotech Eng J SEAGS \& AGSSEA 44(1): 26-33.

Leroueil S, Vaughan PR. 1990. The general and congruent effects of structure in natural soils and weak rocks. Géotechnique 40(3): 467-488.

Leroueil S, Tavenas F, Brucy F, La Rochelle P, Roy M. 1979. Behavior of destructured natural clays. J Geotech Eng 105(6): 759-778.

Lin PS, Lowell CW. 1981. Compressibility of field compacted clay. Joint Research Highway Research, Project report No. C36-5M. Purdue University, $153 \mathrm{p}$.

Liu MD, Carter JP. 1999. Virgin compression of structured soils. Géotechnique 49(1): 43-57.
Liu MD, Carter JP. 2000. Modelling the destructuring of soils during virgin compression. Géotechnique 50(4): 479-483.

Liu MD, Horpibulsuk S, Du YJ. 2015. Framework for the destructuring of clays during compression. Geotech Eng J 46(4): 96-101.

Lloret-Cabot M, Wheeler SJ, Sánchez M. 2017. A unified mechanical and retention model for saturated and unsaturated soil behaviour. Acta Geotech 12(1): 1-21. doi: 10.1007/s11440-016-0497-x.

Low HE, Phoon KK, Tan TS, Leroueil S. 2008. Effect of soil microstructure on the compressibility of natural Singapore marine clay. Rev Can Géotech 45(2): 161-176. doi: 10.1139/T07-075.

Lutenegger AJ, Saber RT. 1988. Determination of collapse potential of soils. Geotech Test $J$ 11(3): 173-178.

McDowell GR. 2002. On the yielding and plastic compression of sand. Soils Found 42(1): 139-145.

McDowell GR, Bolton MD. 1998. On the micromechanics of crushable aggregates. Géotechnique 48(5): 667-679.

Menon M, Jia X, Lair GJ, Faraj PH, Blaud A. 2015. Analysing the impact of compaction of soil aggregates using X-ray microtomography and water flow simulations. Soil Tillage Res 150: 147-157. doi: 10.1016/j.still.2015.02.004.

Mesbah A, Morel JC, Olivier M. 1999. Comportement des sols fins argileux pendant un essai de compactage statique: détermination des paramètres pertinents. Mater Struct 32(9): 687-694.

Mieussens C. 1993. Détermination de la sensibilité des sols aux variations de teneur en eau en laboratoire. Essais à l'oedomètre sur les sols compactés. Projet de méthode LPC, Rapport du LRPC de Toulouse, $12 \mathrm{p}$.

Moussaï B, Didier G, Atlan Y. 1993. Étude d'un matériel de compactage statique et de mesure de la perméabilité des sols fins argileux. Bull Liaison Lab Ponts Chaussées 188: 15-22.

Mun W, McCartney JS. 2015. Compression mechanisms of unsaturated clay under high stresses. Can Geotech J 52: 1-14. doi: 10.1139/cgj-2014-0438.

Nagaraja TS, Srinivasa Murthy BR. 1983. Rationalization of Skempton's compressibility equation. Géotechnique 33(4): 433-443.

Nakata Y, Kato Y, Hyodo M, Hyde AFL, Murata H. 2001. Onedimensional compression behaviour of uniformly graded sand related to single particle crushing strength. Soils Found 41 (2): 39-51. doi: 10.3208/sandf.41.2_39.

Pandian NS, Nagaraj TS, Manoj M. 1997. Re-examination of compaction characteristics of fine-grained soils. Géotechnique 47 (2): 363-366.

Pestana JM, Whittle AJ. 1995. Compression model for cohesionless soils. Géotechnique 45(4): 611-631. doi: 10.1680/ geot.1995.45.4.611.

Qian S, Shi J, Ding JW. 2016. Modified Liu-Carter compression model for natural clays with various initial water contents. Advances in Civil Engineering, 2016, 8 p. doi: 10.1155/2016/ 1691605.

Rücknagel J, Götze P, Hofmann B, Christen O, Marschall K. 2016. The influence of soil gravel content on compaction behaviour and pre-compression stress. Geoderma 209-210: 226-232.

Russell AR. 2011. A compression line for soils with evolving particle and pore size distributions due to particle crushing. Géotech Lett 1: 5-9. doi: 10.1680/geolett.10.00003.

Saad S, Mirzababaei M, Mohamed M, Miraftab M. 2012. Uniformity of density of compacted fibre reinforced clay soil samples prepared by static compaction. Proceeding of 5th European Geosynthetics Congress, Valencia, Spain.

Saffit-Hdadi K, Défossez P, Richard G, Cui YJ, Tang AM. 2009. A method to predict the soil susceptibility to compaction of surface 
layers as a function of water content and bulk density. Soil Tillage Res 105: 96-103.

Schmertmann JH. 1991. The mechanical aging of soils. $J$ Geotech Eng 117(9): 1288-1330.

Seed HB. 1954. Stability and swell pressure characteristics of compacted clays. Clays Clay Miner 3 (1): 483-504. doi: 10.1346/ CCMN.1954.0030140.

Seed HB, Chan CK. 1959. Structure and strength characteristics of compacted clays. J Soil Mech Found Divis, ASCE 85(5): 87-128.

Serratrice JF. 1995a. Comportement d'une argile compactée. Bull Liaison Lab Ponts Chaussées 200: 13-24.

Serratrice JF. 1995b. Comportement d'une craie compactée. Colloquium Mundanum, Craies et schistes, Bruxelles, pp. 1.1.71-1.1.80.

Serratrice JF. 2013. Comportement d'un limon compacté. Bull Lab Ponts Chaussées 280-281: 105-122.

Sharma B, Sridharan A, Talukdar P. 2016. Static method to determine compaction characteristics of fine-grained soils. Geotech Test J. doi: $10.1520 /$ GTJ20150221.

Sharma SS, Ismail MA. 2016. A new normalisation scheme for normal compression and critical state line for soils. Int J Geo-Eng 2016: 7-21. doi: 10.1186/s40703-016-0034-7.

Sheng D, Yao Y, Carter JP. 2008. A volume-stress model for sands under isotropic and critical stress states. Can Geotech $J$ 45: $1639-1645$

Shipton B, Coop MR. 2012. On the compression behaviour of reconstituted soils. Soils Found 52(4), 668-681.

Sivakumar V, Wheeler SJ. 2000. Influence of compaction procedure on the mechanical behaviour of an unsaturated compacted clay, Part 1: Wetting and isotropic compression. Géotechnique 50(4): 359-368.

Skempton AW, Jones OT. 1944. Note on the compressibility of clays. Q J Geol Soc Lond 100: 119-135. doi: 10.1144/GSL. JGS.1944.100.01-04.08.

Skempton AW, Northey RD. 1952. The sensitivity of clays. Géotechnique 2(1): 30-53.

Sun D, Sheng D, Xu Y. 2007. Collapse behaviour of unsaturated compacted soil with different initial densities. Rev Can Géotech 44 (6): 673-686. doi: 10.1139/t07-023.

Tarantino A, De Col E. 2008. Compaction behaviour of clay. Géotechnique 58(3): 199-213. doi: 10.1680/geot.2008.58.3.199.
Terracina F. 1955. Variabilité des caractéristiques d'état des sols. Représentation graphique. Rev Gén Routes Aérodr 279: 61-78.

Terzaghi K. 1927. Principles of final soil classification. Public Roads 8: 41 .

Vaughan PR. 2003. Observations on the behaviour of clay fill containing occluded air bubbles. Géotechnique 53(2): 265-272.

Venkatarama Reddy BV, Jagadish KS. 1993. The static compaction of soils. Géotechnique 43(2): 337-341.

Walker EE. 1923. The properties of powders. Part VI. The compressibility of powders. Trans Faraday Soc 19: 73-82. doi: $0.1039 / \mathrm{tf} 9231900073$.

Wang SY, Chan DH, Lam KC, Au SKA. 2011. Laboratory study of static and dynamic compaction grouting in triaxial condition. Geomech Geoeng 6(1): 9-19.

Wang Y, Cui YJ, Benahmed N, Duc M, Tang AM. 2017. Effects of aggregate size on the compressibility and air permeability of limetreated fine-grained soil. Eng Geol 228: 167-172.

Wu Y, Yamamoto H, Izumi A. 2016. Experimental investigation on crushing of granular material in one-dimensional test. Period Polytech Civil Eng 60(1): 27-36. doi: 10.3311/PPci.8028.

Yaghoubi E, Disfani MM, Arulrajah A, Kodikara J. 2018. Impact of compaction method on mechanical characteristics of unbound granular recycled materials. Road Mater Pavement Des 19(4): 912-934.

Yamamuro JA, Bopp PA, Lade PV. 1996. One dimensional compression of sands at high pressures. J Geotech Eng 122(2): $147-154$

Yang C, Carter JP, Sheng D. 2014. Description of compression behaviour of structured soils and its application. Rev Can Géotech 51(8): 921-933. doi: 10.1139/cgj-2013-0265.

Yoon GL, Kim BT, Jeon SS. 2004. Empirical correlations of compression index for marine clay from regression analysis. Can Geotech J 41(6): 1213-1221.

Zeng LL, Hong ZS, Cui YJ. 2015. Determining the virgin compression lines of reconstituted clays at different initial water contents. Can Geotech J 52(9): 1408-1415.

Zhemchuzhnikov A, Ghavami K, dal Toé Casagrande M. 2015. Static compaction of soils with varying clay content. Key Eng Mater 668: 238. doi: 10.4028/www.scientific.net/KEM.668.238.

Citation de l'article : Jean-François Serratrice. Apport expérimental de la méthode de compactage statique des sols au laboratoire. Rev. Fr. Geotech. 2018, 156, 1 . 